The Detriments of Unmet Sexual Ideals and Buffering Effect of Sexual Communal Strength

Rhonda N. Balzarini ${ }^{1}$, Amy Muise $^{1}$, Kiersten Dobson ${ }^{2}$, Taylor Kohut ${ }^{2}$, Stephanie Raposo ${ }^{1}$, Lorne Campbell ${ }^{2}$

${ }^{1}$ Department of Psychology, York University, Toronto, ON, Canada

${ }^{2}$ Department of Psychology, University of Western Ontario, London, ON, Canada

Correspondence concerning this article should be addressed to Rhonda Balzarini, Department of Psychology Main Office, Behavioural Science Building, York University, Toronto, Ontario, Canada M3J 1P3. E-mail: rbalz@yorku.ca

This work has been supported by an Ontario Trillium Award and Mitacs Accelerate and Elevate Grant awarded to the first author, a Social Science and Humanities Research Council (SSHRC) Insight Grant awarded to Amy Muise, a SSHRC Insight Grant awarded to Lorne Campbell, a SSHRC graduate fellowship awarded to Kiersten Dobson, and a SSHRC graduate fellowship awarded to Stephanie Raposo. 


\begin{abstract}
Despite the importance of sex for the maintenance of satisfying romantic relationships, our understanding of a person's sexual ideals — the traits and attributes a person desires in a sexual partner or experience - and what might buffer against lower satisfaction associated with unmet sexual ideals, is limited. Across four studies including cross-sectional, dyadic, longitudinal, and experimental methods $(N=1,532)$, we draw on the Ideal Standards Model and theories of communal motivation to examine whether unmet sexual ideals are associated with lower sexual satisfaction and relationship quality and test whether higher sexual communal strength - the motivation to meet a partner's sexual needs - buffered these effects. Across studies, when individuals perceived their partner to fall short in meeting their sexual ideals, they reported poorer sexual and relationship quality. People with partners low in sexual communal strength reported poorer sexual satisfaction and relationship quality when their sexual ideals were unmet, but these associations were attenuated among people with partners who were high in sexual communal strength. Perceived partner responsiveness - both in general (Study 2) and to a partner's sexual needs specifically (Study 3) — was one reason why people with partners high in sexual communal strength were buffered against the lower sexual and relational quality associated with unmet ideals.
\end{abstract}

Keywords: romantic relationships; sexual ideals; relationship quality; sexual satisfaction; sexual communal strength 


\section{The Detriments of Unmet Sexual Ideals and Buffering Effect of Sexual Communal Strength}

Sexuality is an important aspect of romantic relationships that differentiates romantic partnerships from other types of close relationships (Schwartz \& Young, 2009). Indeed, sexuality holds such a privileged role in romantic relationships that partners are typically forbidden from seeking sexual interactions with other people outside the relationship (Blanchflower \& Oswald, 2004). For better or worse, romantic partners usually have to rely heavily on each other to fulfill their sexual needs. Unfortunately, sexual incompatibilities (i.e., differences between partners in their sexual preferences and desires) are common and can be distressing in long-term relationships (Miller, Yorgason, Sandberg, \& White, 2003). Different sexual interests between partners are a key reason why couples seek therapy (Beck, 1995; Hawton, Catalan, \& Fagg, 1991; Henry \& Miller, 2004; Miller et al., 2003; Rosen, 2000) and are among the most difficult types of concerns to successfully resolve (Sanford, 2003). Despite the prevalence of sexual incompatibilities in relationships, our understanding of a person's sexual ideals (i.e., the traits and attributes a person desires in a sexual partner and the characteristics of the sexual experience they hold to be ideal) and what might mitigate dissatisfaction associated with unmet sexual ideals remains limited. In the current research, we draw on theories of ideal standards in relationships (e.g., Fletcher, Simpson, \& Thomas, 2000; Fletcher, Simpson, Thomas, \& Giles, 1999; Simpson, Fletcher, \& Campbell, 2001) and theories of sexual communal motivation (e.g., Muise \& Impett, 2016) to examine whether perceptions of unmet sexual ideals in a relationship are associated with lower sexual satisfaction and relationship quality, and if so, whether sexual communal strength — being attuned to and motivated to meet a partner's sexual needs — can buffer against the lower sexual and relationship quality predicted to be associated with unmet sexual ideals.

\section{Ideal Standards for a Romantic Relationship}


Ideal standards for a romantic relationship reflect one's hopes and expectations for a romantic partner (Fletcher et al., 1999). A substantial amount of theoretical and empirical work has focused on what individuals are looking for in potential romantic partners (for a review see Campbell \& Stanton, 2014, or Eastwick, Luchies, Finkel, \& Hunt, 2014; also see Buss, 1999; Eastwick, Finkel, \& Simpson, 2019; Fletcher, Overall, \& Campbell, 2019; Fletcher, Simpson, Campbell, \& Overall, 2013; Simpson, Fletcher, \& Campbell, 2001). This work has been informed by the Ideal Standards Model (Fletcher et al., 1999; Simpson et al., 2001), which posits that people possess images of their ideal romantic partner across a range of attributes (e.g., interpersonal warmth, physical appeal, status and resources), and these ideal preferences are used to evaluate both potential mates and actual partners in existing relationships. According to the Ideal Standards Model, relationships should develop and be maintained more smoothly and successfully when individuals enter relationships with a partner who more closely matches their ideal preferences (Campbell, Overall, Rubin, \& Lackenbauer, 2013; Campbell, Simpson, Kashy, \& Fletcher, 2001; Csajbók \& Berkics, 2017; Fletcher et al., 2000; Overall, Fletcher, \& Simpson, 2006). Accumulating research supports this claim (Campbell, Chin, \& Stanton, 2016; Fletcher et al., 2019; Gerlach, Arslan, Schultze, Reinhard, \& Penke, 2019).

Past research has examined discrepancies between a person's ideal standards and their perceptions of their current partner or relationship meeting their standards. Reviews of the literature have revealed that different methods of assessing ideal-partner discrepancy yield different findings, and the match between a persons' ideals and their partner's traits is not always associated with relationship initiation or quality (for a review see Campbell \& Stanton, 2014; or Eastwick et al; 2014). However, a person's perception of the extent to which a partner meets their ideals is consistently associated with relationship satisfaction (e.g., Buyukan-Tetik, 
Campbell, Finkenauer, Karremans, \& Kappen, 2017; Campbell et al., 2001; Fletcher et al., 1999; Fletcher et al., 2000; Kelley \& Burgoon, 1991; Meltzer, McNulty, Jackson, \& Karney, 2014) and the likelihood of the relationship enduring (see Campbell et al., 2019; Gerlach et al., 2019). In the current work, we were interested in this specific facet of ideal standards, namely, individuals' perceptions of their partner meeting their sexual ideals. This approach is consistent with the key hypothesis of the Ideal Standards Model (Fletcher et al., 1999; Simpson et al., 2001), that suggests that romantic outcomes (e.g., partner selection, partner evaluation, breakup) should be associated with the degree to which a person perceives their partner to meet their ideals, and Interdependence Theory (Kelley \& Thibault, 1978; Rusbult \& Buunk, 1993) that posits that past experiences set comparison levels for people and that if met, satisfaction is higher. That is, if a partner exceeds a person's comparison level for an ideal sexual partner and ideal sexual experiences, we would expect the person to report that their partner meets their sexual ideals, which in turn is associated with greater sexual and relationship quality. Accordingly, sexual and relationship quality should be associated with the degree to which a person perceives their partner to meet their ideals or to exceed their comparison levels for an ideal sexual partner.

\section{Ideal Standards for a Sexual Relationship}

Although psychologists have devoted ample attention to understanding whether ideal standards for a romantic partner are associated with relationship and sexual quality, little attention has been paid to the ideal standards people hold for their sexual relationships. To date, research has assessed how relationship ideals shape outcomes related to seeking out, attracting, and retaining romantic partners, but there is currently a paucity of measures designed to assess individuals' trait variation in sexual ideals. There is also a lack of information about what one's sexual ideals would encompass and whether unmet sexual ideals shape sexual and relationship 
outcomes. Previous research on sexual discrepancies has predominately focused on differences in desired frequency of sex, levels of sexual desire, and specific sexual acts that are commonly engaged in (e.g., oral sex, vaginal intercourse, kissing), without simultaneously assessing the extent to which these feelings and behaviors, as well as broader sexual interests and preferences, are actually characteristic of a person's ideal preferences in a sexual relationship.

While past work has not investigated unmet sexual ideals specifically, differences between partners in their levels of sexual desire or in their preferences for specific sexual behaviors (e.g., sexual intercourse, use of porn) are common (Davies, Katz, \& Jackson, 1999; Dworkin \& O’Sullivan, 2005; Kohut, Balzarini, Fisher, \& Campbell, 2018; Impett \& Peplau, 2003; Mark, 2012; Mark \& Murray, 2012; Miller \& Byers, 2004; Risch et al., 2003; Santilla et al., 2007) and tend to be associated with lower sexual satisfaction and poorer relationship quality (Davies et al., 1999; Kohut et al., 2018; Mark, 2012; Mark \& Lasslo, 2018; Santilla, et al., 2007; Willoughby, Farero, \& Busby, 2014; Zilbergeld \& Ellison, 1980), although some findings are mixed or not consistently replicated (e.g., Mark, 2014; Mark \& Murray, 2012; Rosen, Bailey, \& Muise, 2018; Sutherland, Rehman, Fallis, \& Goodnight, 2015; Willoughby \& Vitas, 2012). Further, given that both cross-sectional (e.g., Butzer \& Campbell, 2008; Cupach \& Comstock, 1990; Dundon \& Rellini, 2010; Litzinger \& Gordon, 2005; Webster, Laurenceau, et al., 2015) and longitudinal studies (e.g., Byers, 2005; McNulty \& Fisher, 2008; Sprecher, 2002; Yeh, Lorenz, Wickrama, Conger, \& Elder, 2006) indicate that a person's satisfaction with their sex life is associated with their overall feelings of satisfaction with, and commitment to, their romantic relationship, sexual problems stemming from unmet sexual ideals may extend beyond the bedroom. 
Given the previous research highlighting the typically negative effects of sexual discrepancies, and similar to research on ideal standards for a romantic relationship, we expect that individuals' perceptions of a partner meeting their sexual ideals would be associated with their evaluations of their sexual and relationship quality. Specifically, we expect that, in line with the Ideal Standards Model (Fletcher et al., 1999), when people perceive that their partner does not meet their sexual ideals, they will report lower sexual satisfaction and relationship quality.

\section{Sexual Communal Strength}

If, in fact, unmet sexual ideals are common in relationships and are associated with lower sexual satisfaction and relationship quality, what might mitigate negative associations with unmet sexual ideals? The motivation to care for and be responsive to a partner's needs (Clark \& Mills, 2012) may be one factor, as it appears to be a crucial component of satisfying interpersonal relationships and personal well-being (for a review, see Le, Impett, Lemay, Muise, \& Tskhay, 2018). People who are motivated to be responsive to their partner's needs noncontingently — those high in communal strength — tend to be better at navigating situations of conflicting interests and are more satisfied in their relationships, even when they have different interests or when they sacrifice their own preferences for their partner (see Kogan et al., 2010). Indeed, above and beyond general communal strength, people high in sexual communal strength - those who are attuned to and motivated to meet their partner's sexual needs — report higher sexual desire and satisfaction over the course of their relationship (Muise et al., 2013; Day et al., 2015), and have partners who report greater satisfaction with and commitment to their relationships (Muise \& Impett, 2015).

In the current research we sought to investigate whether sexual communal strength might buffer against the negative consequences of unmet sexual ideals. Past research has shown that 
people who are highly motivated to meet their partners' sexual needs are more successful at navigating situations of sexual desire discrepancies. In these situations, people higher in sexual communal strength tend to maintain sexual satisfaction even on days when they engage in sex but have different levels of desire than their partner (Day et al., 2015). Importantly, in this past work, sexually communal people still experienced sexual differences with their partner, but they were able to navigate these situations in ways that maintained both partners' satisfaction. This could be, in part, because sexual communal strength is associated with a greater understanding of partners having different sexual interests. For example, people higher in sexual communal strength are more understanding and caring when a partner is not in the mood for sex and they tend to feel less resentment in response to a partner declining their sexual advances (see Kim, Muise, \& Impett, 2018). Even among couples coping with extenuating factors that influence their sexual interests and desire (e.g., transitioning to parenthood; Muise, Kim, Impett, \& Rosen, 2017) and those coping with clinical sexual issues (e.g., low sexual desire; Hogue, Rosen, Bockaj, Impett, \& Muise, 2019; sexual pain; Muise, Bergeron, Impett, \& Rosen, 2017), when one partner is higher in sexual communal strength, both partners reported higher sexual and relationship satisfaction.

Having a partner who is high in sexual communal strength might be particularly important for buffering against the negative consequences of unmet sexual ideals in relationships because sexually communal people are more motivated to provide benefits to their partner and less motivated to avoid costs to the self in pursuit of meeting their partner's needs (Day et al., 2015). Therefore, having unmet sexual ideals may be less consequential for people with partners who are higher in sexual communal strength given that the partner is highly motivated to demonstrate responsiveness. In related work on sacrifice-giving up one's 
own interest for a partner or relationship_-people who perceived their partners as more responsive to their needs tended to view a sacrifice more favorably and were more satisfied after making a sacrifice compared to people with less responsive partners, and this was true even for large sacrifices, such as moving to a new city for a partner's career, that are objectively costly (Visserman, Muise, Righetti, Horne, \& Impett, in prep). In fact, the association between partner differences (i.e., situations of conflicting interests or sacrifice) and satisfaction differs based on a partner's motivation to provide support and meet the person's needs. People who are motivated to meet their partner's needs tend to be able to maintain satisfaction in situations of conflicting interest (Impett, Peplau, \& Gable, 2005), but if a partner is unsupportive, conflicting interests are associated with lower satisfaction (Wei-Fang \& TsuiShan, 2017). Research also indicates that when people feel their partners are responsive, they are more inclined to invest in their relationships (Murray, Holmes, \& Griffin, 1996) and to perceive poorer quality of alternatives to the relationship (Segal \& Fraley, 2016). This line of research presents evidence that one reason why having a partner higher in sexual communal strength might buffer lower sexual and relationship quality from unmet sexual ideals is because people perceive communal partners as more responsive, even when they do not meet their ideals.

\section{Perceived Partner Responsiveness}

Partners who are high in sexual communal strength tend to be perceived as more responsive (Muise \& Impett, 2015) and this might be one reason why having a sexually communal partner buffers people from lower satisfaction in response to unmet sexual ideals. Perceived partner responsiveness reflects the extent to which individuals believe their relationship partner understands, validates, and cares for them (Reis, 2007). Not only are people higher in sexual communal strength perceived as more responsive during sex specifically (Muise 
\& Impett, 2015), but they are also more generally responsive to a partner's needs (see Muise \& Impett, 2016 for a review). Given that people higher in sexual communal strength are more motivated to meet their partner's needs even when those needs are different than their own (Day et al., 2015), communal partners are likely to be perceived as responsive even when the person has unmet sexual needs.

Perceiving a partner as highly responsive is associated with greater satisfaction, and commitment (Birnbaum, Reis, Mizrahi, Kanat-Maymon, Sass, \& Granovski-Milner, 2016; Reis et al., 2004; Segal \& Fraley, 2016) and helps couples more successfully navigate conflicts of interest (Impett, Gable, \& Peplau, 2005; Wei-Fang \& Tsui-Shan, 2017; Visserman et al., in prep) and maintain satisfaction in the face of relationship conflict (Gordon \& Chen, 2016). When couples face conflict or challenges in their relationship, perceiving a partner as highly responsive can encourage better coping and increase the amount of support provided by enabling experiences of closeness and open communication during the event (Manne et al., 2018). Perceived partner responsiveness also tends to make it safe for people to reveal their needs and vulnerabilities to their partners (Clark \& Lemay, 2010; Laurenceau, Barrett, \& Pietromonaco, 1998) and can help promote confidence that a partner is willing to provide responsive support (see Reis, Lemay, \& Finkenauer, 2017). Given the previous research showing that people high in sexual communal strength are perceived as more responsive to their partner's needs and that perceived partner responsiveness is associated with greater satisfaction and commitment, we explored whether perceived partner responsiveness (both in general and to sexual needs specifically) is one reason why having a partner who is high in sexual communal strength buffers the detriments of unmet sexual ideals.

\section{Overview of the Current Research}


In the current research, we draw on the Ideal Standards Model to investigate sexual ideals in relationships. In line with past research, we predicted that unmet sexual ideals would be associated with lower sexual satisfaction and relationship quality. In addition, we draw on theories of communal motivation to examine whether sexual communal strength buffers the detriments of unmet sexual ideals. Our key prediction is that individuals who are high in sexual communal strength or who have a partner who is high in sexual communal strength, would be buffered against experiencing lower sexual satisfaction and relationship quality in the face of unmet sexual ideals. We further sought to assess whether one of the mechanisms for why having a partner higher in sexual communal strength might buffer against lower sexual and relationship quality associated with unmet ideals is because communal partners are perceived as more responsive.

We examined our predictions in four pre-registered studies that employ a variety of methods, including cross-sectional, daily experience sampling, longitudinal, and experimental designs. In Study 1, a cross-sectional study of long-term romantic couples, we test our key predictions that sexual communal strength buffers against lower sexual satisfaction and relationship quality associated with unmet sexual ideals. In Studies 2 and 3, couples completed a 21-day daily diary. In these two daily experience studies, we examine how daily changes (e.g., within-person fluctuations) in people's sexual ideals being met are associated with daily sexual satisfaction and relationship quality, and test whether people who are higher in sexual communal strength, or who have sexually communal partners, are buffered against experiencing lower sexual and relationship quality on days when they report unmet sexual ideals compared to less sexually communal people. We further assess whether having a partner higher in sexual communal strength buffers against lower sexual and relationship quality associated with unmet 
ideals, in part, because sexually communal partners are perceived as more responsive — both generally (Study 2) and specifically in the sexual domain (Study 3). In Study 3, we also include a three-month longitudinal follow-up survey to assess associations between unmet sexual ideals and satisfaction and the buffering effect of sexual communal strength over time. In Study 4, we experimentally manipulate both perceptions of a partner's sexual communal strength and met versus unmet sexual ideals in a current relationship to provide causal evidence for the deleterious effects of unmet sexual ideals in relationships, and the buffering effect of perceiving a partner as high in sexual communal strength.

Given that sexual frequency (Call, Sprecher, \& Schwartz, 1995; Muise, Schimmack, \& Impett, 2016) and desire (Muise et al., 2019) are associated with sexual and relationship quality, and sexual frequency (Call et al., 1995) and desire (Klusmann, 2002; McNulty, Wenner, \& Fisher, 2016; Sprecher, 2002) tend to fade overtime, we also sought to isolate the effects of unmet ideals to show that the effects are about having unmet sexual ideals specifically, and not just driven by low sexual frequency or desire. More specifically, in Studies 2 and 3, we examined whether the effects remained significant after controlling for sexual frequency and sexual desire.

\section{Study 1}

In Study 1, we test our two key predictions in a cross-sectional study of romantic couples. First, we test whether people who report greater unmet sexual ideals report lower sexual and relationship quality compared to people with met ideals. Next, we test whether people higher in sexual communal strength, or who have partners higher in sexual communal strength (compared to lower sexual communal strength), would be buffered against the negative associations between unmet sexual ideals and sexual and relationship quality. We also explored gender as a 
potential moderator of the association between unmet sexual ideals and sexual satisfaction and relationship quality and examined whether gender influenced the buffering effect of sexual communal strength. The hypotheses were pre-registered on the OSF.

\section{Method}

\section{Participants and Procedure}

We recruited mixed-sex couples from the United States and Canada through Qualtrics Panel, an online crowdsourcing platform that is commonly used for psychological research. To enter the study, we required couples to be involved in a romantic relationship of at least four months, for both individuals in the relationship to be willing to participate, for participants to be at least 18 years of age, and for participants to meet standard instruction and attention checks. We aimed to recruit at least 200 couples, a number that far exceeds recommendations for achieving sufficient power with dyadic data (see Kenny, Kashy, \& Cook, 2006 recommendation for 100 couples). A total of 267 couples accessed the online study, and based on the preregistered criteria for inclusion, of those, 60 were removed because one or both partners failed one of four attention or instruction checks $(22.47 \%) .{ }^{1}$ Our final sample consisted of 207 couples. Post-hoc power analyses using an online application for calculating power curves for APIM models with indistinguishable dyads (Ackerman, Ledermann, \& Kenny, in prep; Kenny \& Ackerman, 2019) indicated that with 207 couples, we had 99\% power to detect a small effect. Participants were mostly married (88.4\%), Caucasian (84.5\%), and monogamous (88.9\%). Most participants were in their mid-40's $(M=45.80$ years, $S D=10.34)$ and were in long-term

\footnotetext{
1 As widely employed in the literature (e.g., Berinsky, Margolis, \& Sances, 2014; Curran, 2016), all studies used attention check questions, wherein we asked participants to select a particular answer choice for that question (e.g., "I am paying attention to this survey. If you are paying attention, select number three."). We made the decision a priori to exclude participants who did not select the instructed value.
} 
relationships with their partner $(M=17.32$ years, $S D=10.23)$. See Table 1 for more information about the demographics.

Table 1

Demographic Information for all Studies

\begin{tabular}{lcccc}
\hline & Study 1 & Study 2 & Study 3 & Study 4 \\
\hline Age - Mean (SD) & $45.80(10.3)$ & $30.70(8.96)$ & $32.63(10.2)$ & $34.80(15.2)$
\end{tabular}

Gender

$\begin{array}{lllll}\text { Male } & 207(50 \%) & 118(50 \%) & 115(47.5 \%) & 206(33.3 \%) \\ \text { Female } & 207(50 \%) & 118(50 \%) & 124(51.2 \%) & 408(66.0 \%) \\ \text { Other } & - & - & 2(1.3 \%) & 4(0.70 \%)\end{array}$

Race

$\begin{array}{lllll}\text { White } & 350(82.9 \%) & 172(72.9 \%) & 158(65.6 \%) & 560(90.6 \%) \\ \text { Asian } & 9(2.1 \%) & 40(16.9 \%) & 38(15.7 \%) & 22(3.4 \%) \\ \text { Black } & 28(6.6 \%) & 5(2.1 \%) & 11(4.5 \%) & 10(1.6 \%) \\ \text { Hispanic } & 19(4.5 \%) & 13(5.5 \%) & 10(4.1 \%) & 6(1.0 \%) \\ \text { Am. Indian } & 2(0.5 \%) & 4(1.7 \%) & - & 1(0.2 \%) \\ \text { Bi-racial } & - & - & 14(5.8 \%) & 16(2.6 \%) \\ \text { Other } & 6(1.4 \%) & 6(2.5 \%) & 10(4.1 \%) & 4(0.6 \%)\end{array}$

Sexual Orientation

$\begin{array}{lllll}\text { Heterosexual } & 414(100 \%) & 209(88.6 \%) & 197(81.4 \%) & 535(86.6 \%) \\ \text { Lesbian/Gay } & - & - & 8(3.3 \%) & 17(1.8 \%) \\ \text { Bisexual } & - & 25(10.6 \%) & 22(9.1 \%) & 50(8.1 \%) \\ \text { Other } & - & 2(0.8 \%) & 15(6.2 \%) & 5(0.8 \%)\end{array}$




\begin{tabular}{lllll} 
Relationship Status & & & & \\
Dating & $26(6.2 \%)$ & $98(41.5 \%)$ & $107(44.1 \%)$ & $293(47.4 \%)$ \\
Engaged & $22(5.2 \%)$ & $27(11.4 \%)$ & $19(7.9 \%)$ & $37(6.0 \%)$ \\
Married & $366(86.7 \%)$ & $104(44.1 \%)$ & $113(47.1 \%)$ & $284(46.0 \%)$ \\
Other & - & $7(3.0 \%)$ & $3(1.2 \%)$ & $4(0.6 \%)$ \\
\hline
\end{tabular}

Eligible participants completed an online survey that asked both partners to report on the extent to which their partner met their sexual ideals, in addition to their sexual communal strength, sexual satisfaction, relationship satisfaction, and commitment. Additional measures were included for other purposes and will not be discussed further in the current paper. For a complete listing of the measures, please see the OSF. Participants could earn up to $\$ 10.50$ (USD) per person for their participation.

\section{Measures}

Extent Partner Meets Sexual Ideals. Across three pilot studies, we developed a measure to assess the extent to which people perceive their partner as meeting their sexual ideals (see supplemental materials). The Sexual Ideal Scale includes 30 items asking participants to indicate the extent to which their partner met their sexual ideals on a variety of traits and sexual preferences (30 items, e.g., "My partner engages in oral sex with me as much I want my ideal sexual partner to"). Participants were asked to respond to each item using a 7-point Likert scale $(1=$ does not meet my ideal, $7=$ completely meets my ideal $)$. The items were reverse coded and then mean aggregated across ideals to create an aggregate score, with higher scores indicating greater unmet sexual ideals $(\alpha=.93 ; M=2.28, S D=1.03)$. For more information about the scales development and final items, please see the supplemental materials or OSF. 
Sexual Satisfaction. The Global Measure of Sexual Satisfaction (five items; GMSEX;

Lawrance \& Byers, 1998) was used to assess sexual satisfaction. Participants were asked to indicate on 7-point bipolar scales which best described their current sexual relationship: unsatisfying-satisfying, unpleasant-pleasant, good-bad, negative-positive, and worthlessvaluable. Items were mean aggregated, with higher scores indicating higher sexual satisfaction $(\alpha=.97 ; M=5.94, S D=1.39)$. Lawrance and Byers (1998) provided evidence for the reliability and validity of the GMSEX.

Relationship Satisfaction. The Investment Model Scale (IMS; Rusbult, Martz, \& Agnew, 1998) was used to assess relationship satisfaction (three items: e.g., "I feel satisfied with our relationship"). Possible responses were on a 9-point scale ( $1=$ do not agree at all, $9=$ agree completely), and the items were mean aggregated, with higher scores indicating more satisfaction $(\alpha=.95 ; M=7.45, S D=1.96)$. Rusbult and colleagues (1998) provided evidence for the reliability and validity of the IMS, which includes subscales for relationship satisfaction and commitment.

Commitment. The IMS (Rusbult et al., 1998) was used to assess commitment to the relationship (four items: e.g., "I am committed to maintaining my relationship with my partner”). Possible responses were on a 9 -point scale $(1=$ do not agree at all, $9=$ agree completely $)$, and the items were mean aggregated, with higher scores indicating greater commitment $(\alpha=.95 ; M=$ $8.29, S D=1.39)$.

Sexual Communal Strength. The Sexual Communal Strength Scale (six items; Muise et al., 2013) was used to assess the degree to which a partner is attuned to and motivated to meet their partner's sexual needs. Participants completed the measure by rating items such as, "How far would you be willing to go to meet your partner's sexual needs?” on a 5-point Likert scale (1 
$=$ strongly disagree, $5=$ strongly agree . Items were reverse coded when necessary, and were mean aggregated, with higher scores indicating higher sexual communal strength $(\alpha=.78, M=$ 3.93, $S D=0.70$ ). Muise and colleagues (2013) provided evidence for the reliability and validity of the Sexual Communal Strength Scale (also see Muise \& Impett, 2019).

\section{Results}

\section{Unmet Sexual Ideals and Sexual and Relationship Quality}

First, we tested whether the extent to which a partner meets one's sexual ideals was associated with both partners' relationship quality with a linear mixed modeling approach guided by the actor-partner independence model (APIM; Kenny et al., 2006). The initial model involved the prediction of an outcome (either sexual satisfaction, relationship satisfaction, or commitment) using actors' and partners' ratings of the extent to which their partner met their sexual ideals. In line with our pre-registered predictions, the results of the APIM analyses showed that when people reported that their partner did not meet their sexual ideals, they reported feeling less satisfied with their sexual $(b=-.47, t(409)=-8.45, p<.001,95 \%$ CI $[-.58,-.36])$ and romantic relationship $(b=-.77, t(409)=-10.14, p<.001,95 \%$ CI $[-.92,-.62])$, and being less committed to maintaining their relationship $(b=-.41, t(406)=-6.77, p<.001,95 \% \mathrm{CI}[-.52,-.29])$. Additionally, when people reported that their partner did not meet their sexual ideals, their partner also reported lower sexual $(b=-.29, t(409)=-.5 .16, p<.001,95 \% \mathrm{CI}[-.40,-.18])$ and relationship satisfaction $(b=-.37, t(409)=-.4 .92, p<.001,95 \%$ CI $[-.52,-.22])$, and being less committed to maintaining their relationship $(b=-.36, t(406)=-.5 .98, p<.001,95 \%$ CI $[-.48,-$ $.24])$.

\section{Moderating Role of Sexual Communal Strength}


Next, as our primary goal was to test whether a person's perception of the extent to which their partner meets their sexual ideals was associated with lower sexual satisfaction, relationship satisfaction, and commitment, and whether this was moderated by their own and their partner's sexual communal strength, we tested whether sexual communal strength buffered against the negative associations between unmet sexual ideals and sexual and relationship quality. In a separate model, we assessed whether sexual communal strength moderated the effects (Moderated APIM; see Garcia, Kenny, \& Lederman, 2015) by adding to the model both partners' sexual communal strength, and the interaction between both partners' sexual communal strength and both partners' ratings of their partner meeting their sexual ideals. That is, the main effects in the model included actor unmet sexual ideals, partner unmet sexual ideals, actor sexual communal strength, and partner sexual communal strength. Further, there were interactions between actor unmet sexual ideals and actor sexual communal strength, actor unmet sexual ideals and partner sexual communal strength, partner unmet sexual ideals and actor sexual communal strength, and partner unmet sexual ideals and partner sexual communal strength. Importantly, we pre-registered the prediction that the interaction between an actor's unmet sexual ideals and the actor and partner's sexual communal strength would buffer the actor's sexual and relationship quality, but we had no expectations about the interaction of the partner's effects, and thus these findings can be found in the Supplemental Materials. When an interaction was significant, we tested simple effects at high (one standard deviation above) and low (one standard deviation below) levels of sexual communal strength (Aiken, West, \& Reno, 1991). The 
$\underline{\text { analytic plan and the data and syntax }}{ }^{2}$ for all analyses reported for this study can be found on the OSF. Correlations between all variables are presented in Table 2.

Table 2

Correlations Among Focal Variables for Study 1

\begin{tabular}{lrrrrr}
\hline & $\mathbf{1}$ & $\mathbf{2}$ & $\mathbf{3}$ & $\mathbf{4}$ & $\mathbf{5}$ \\
\hline 1. Rel Satisfaction &. $\mathbf{7 5} * * *$ & & & & \\
2. Sexual Satisfaction & $.81 * * *$ &. $\mathbf{7 4 * * *}$ & & & \\
3. Commitment & $.73 * * *$ & $.63 * * *$ &. $\mathbf{6 3} * * *$ & & \\
4. SCS & $.42 * * *$ & $.43 * * *$ & $.47 * * *$ &. $\mathbf{3 4 * * *}$ \\
5. Unmet Ideals & $-.52 * * *$ & $-.48 * * *$ & $-.46 * * *$ & $-.30 * * *$ & $\mathbf{. 6 0} * * *$ \\
& & & & & \\
\hline
\end{tabular}

Note: ${ }^{* * *} p<.001, * * p<.01,{ }^{*} p<.05$. Correlations between partners are bolded on the diagonal, with actor's correlations below the diagonal. Rel satisfaction = relationship satisfaction; $\mathrm{SCS}=$ sexual communal strength. The correlation between an individual's unmet sexual ideals and their partner's sexual communal strength was, $r=-.41, p<.001$.

The results of the analyses suggest that both actor and partner sexual communal strength moderated associations between actors' unmet sexual needs and indicators of sexual and relationship quality, though partners' sexual communal strength appeared to do so more consistently (see Table 3 and Figures 1A-1C). Specifically, people with partners who were low in sexual communal strength reported lower sexual satisfaction $(b=-.50, t(358)=-6.72, p<$ $.001,95 \%$ CI $[-.65,-.35])$, relationship satisfaction $(b=-.89, t(342)=-8.26, p<.001,95 \%$ CI [$1.10,-.68])$, and commitment $(b=-.37, t(387)=-5.08, p<.001,95 \%$ CI $[-.52,-.23])$ when they

\footnotetext{
${ }^{2}$ Note that the data and syntax for all studies are currently in private components that will be made available upon acceptance of the paper. All other components are public for review.
} 
reported higher unmet sexual ideals. However, the association between unmet sexual ideals and sexual and relationship quality were reduced to non-significance (sexual satisfaction: $p=.646$; commitment: $p=.099$ ) or were attenuated (relationship satisfaction: $b=-.27, t(393)=-2.19, p=$ $.03,95 \%$ CI $[-.51,-.03])$ among people who had partners who were higher in sexual communal strength. As such, people who had partners who were higher in sexual communal strength were buffered from the negative associations between unmet sexual ideals and sexual and relationship quality.

Table 3

APIM Models with Reports of Unmet Sexual Ideals (USI) and Sexual Communal (SCS) Strength Predicting Sexual and Relationship Quality in Study 1

\begin{tabular}{|c|c|c|c|c|c|c|}
\hline & \multirow{2}{*}{\multicolumn{2}{|c|}{$\begin{array}{l}\text { Relationship } \\
\text { Satisfaction }\end{array}$}} & \multirow{2}{*}{\multicolumn{2}{|c|}{$\begin{array}{c}\text { Sexual } \\
\text { Satisfaction }\end{array}$}} & \multirow{2}{*}{\multicolumn{2}{|c|}{ Commitment }} \\
\hline & & & & & & \\
\hline & $\boldsymbol{b}$ & $S E$ & $b$ & $S E$ & $\boldsymbol{b}$ & $S E$ \\
\hline \multicolumn{7}{|l|}{ Fixed Effects } \\
\hline Intercept & 7.61 & $.10 * * *$ & 6.11 & $0.07 * * *$ & 8.50 & $.06 * * *$ \\
\hline Actor (A) USI & -.58 & $.08 * * *$ & -.27 & $0.06 * * *$ & -.26 & $.06 * * *$ \\
\hline Partner (P) USI & -.16 & $.08 *$ & -.10 & .06 & -.13 & $.06 *$ \\
\hline A SCS & .53 & $.11 * * *$ & .36 & $.08 * * *$ & .44 & $.08 * * *$ \\
\hline P SCS & .42 & $.11 * * *$ & .48 & $.08 * * *$ & .20 & $.08 * *$ \\
\hline A USI x A SCS & -.22 & .12 & -.07 & .08 & .22 & $.08 * *$ \\
\hline A USI x P SCS & .45 & $.23 * * *$ & .33 & $.08 * * *$ & .16 & $.08 *$ \\
\hline P USI x A SCS & .46 & $.12 * * *$ & .39 & $.08 * * *$ & .41 & $.08 * * *$ \\
\hline P USI x P SCS & -.25 & $.12 *$ & -.13 & .08 & -.03 & .08 \\
\hline
\end{tabular}


Note: ${ }^{* * *} p<.001,{ }^{* *} p<.01,{ }^{*} p<.05$. Also note, the main effect coefficients differ slightly from those reported in-text values because the in-text values are from the main effects only models and the values in the table are from the full model with interactions included.

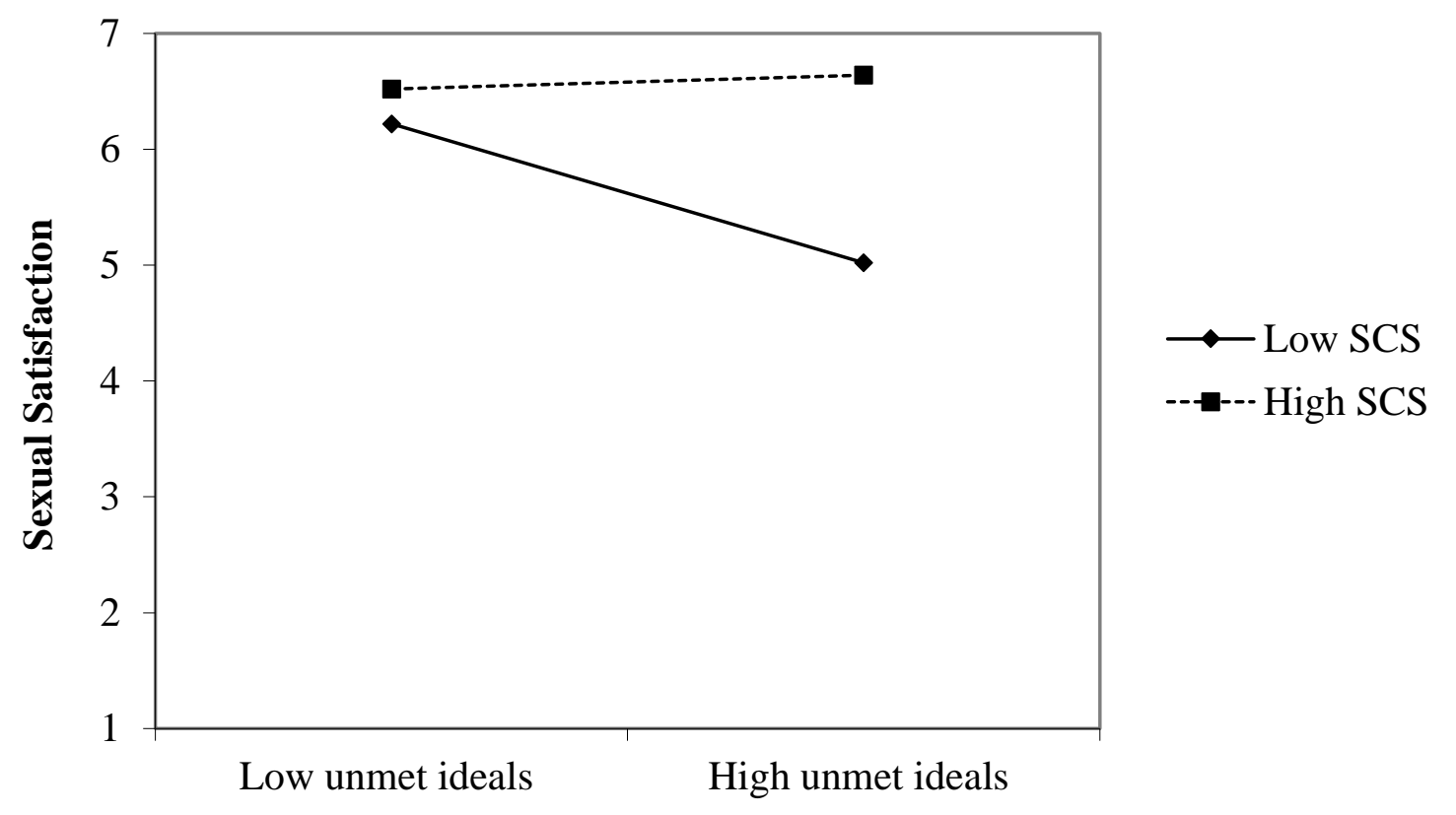

Fig. 1A. The interactive effect of actor's unmet sexual ideals and partner's sexual communal strength (SCS) on actor's sexual satisfaction (Study 1). 


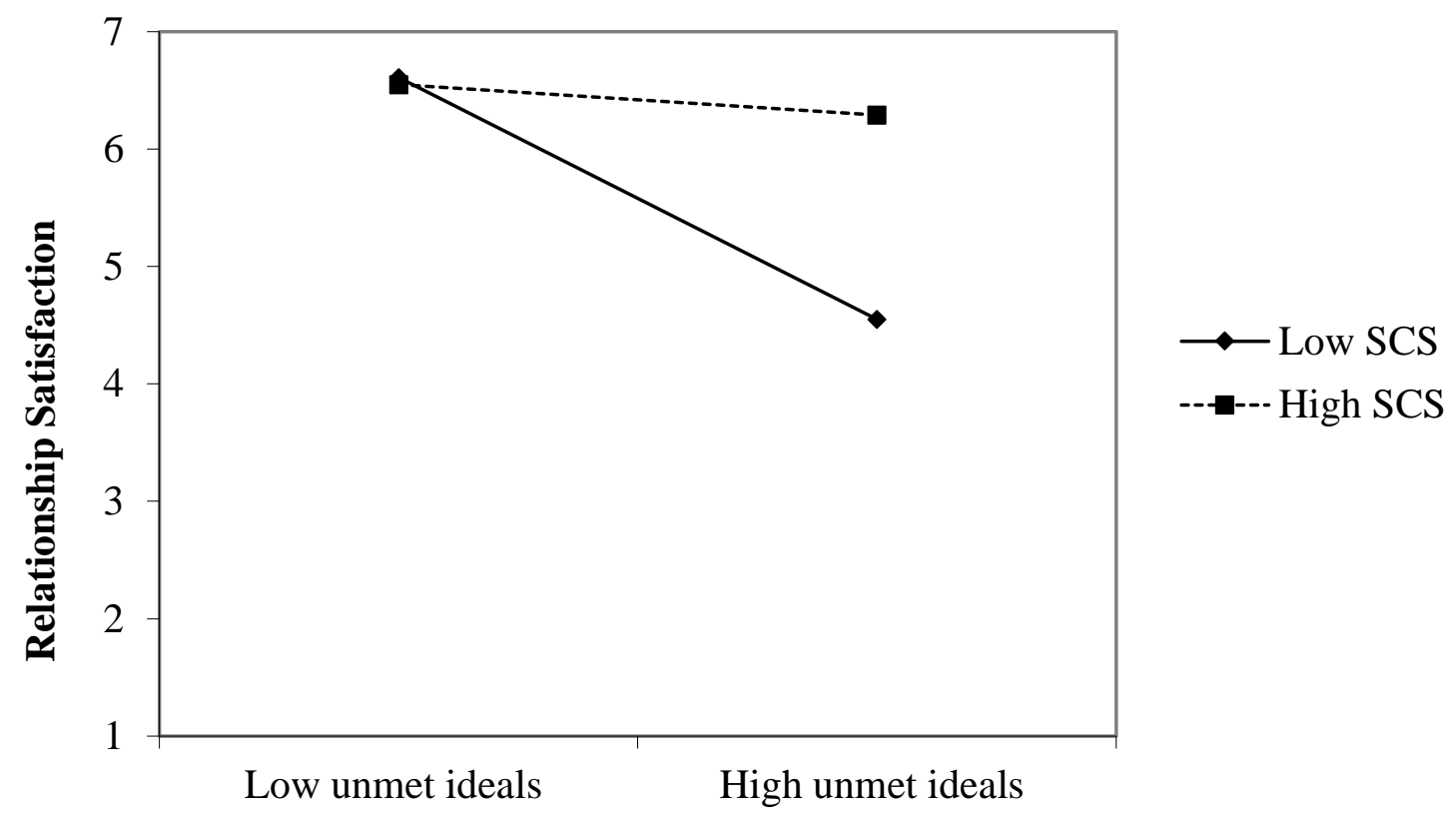

Fig. 1B. The interactive effect of actor's unmet sexual ideals and partner's sexual communal strength (SCS) on actor's relationship satisfaction (Study 1).

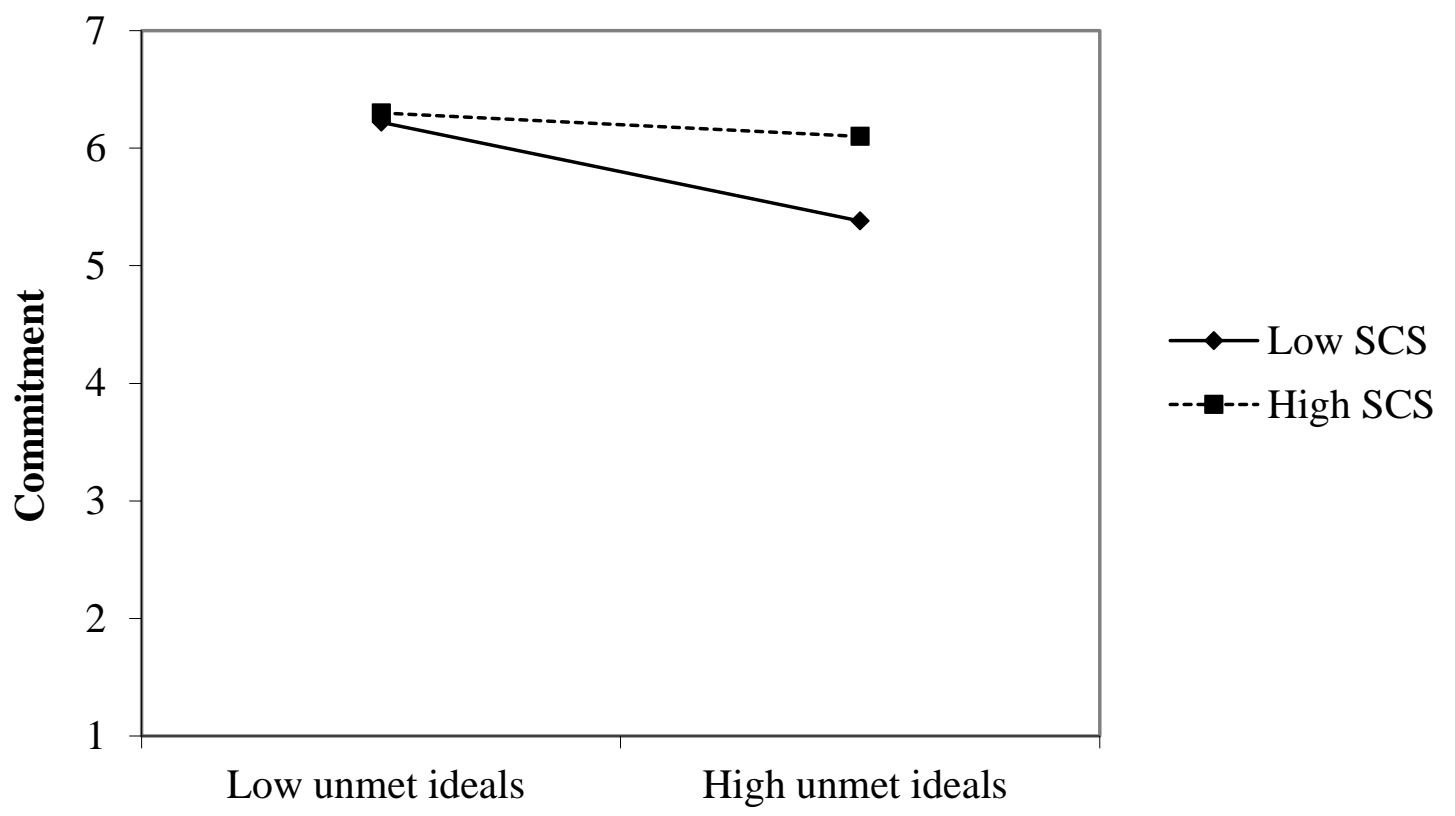

Fig. 1C. The interactive effect of actor's unmet sexual ideals and partner's sexual communal strength (SCS) on actor's commitment (Study 1). 
In contrast, of the three interaction effects predicted for actor's sexual communal strength, only the association between unmet sexual ideals and commitment was significantly moderated by a person's own sexual communal strength (see Table 3). That is, for people who were low in sexual communal strength, unmet sexual ideals were associated with feeling significantly lower commitment to the relationship $(b=-.41, t(387)=-5.63, p<.001,95 \%$ CI $[-$ $.55,-.27])$, whereas the association between unmet sexual ideals and commitment was reduced to non-significance among individuals who were high in sexual communal strength $(p=.242)$. Importantly, and contrary to our predictions, the association between unmet sexual ideals and a person's own sexual $(p=.389)$ and relationship $(p=.069)$ satisfaction were not moderated by their own sexual communal strength. Partner interactions (e.g., the effects of a partner's unmet ideals and the individual's or the partner's sexual communal strength) can be found in the Supplemental Materials.

\section{Providing Evidence for Generalizability of the Findings}

Next, given the extensive literature on gender differences in sexuality in relationships (see review by Peplau, 2003; Petersen \& Hyde, 2010), across the studies, we conducted exploratory analyses to test whether men versus women were more impacted by unmet sexual ideals, and whether gender moderated the key interaction between unmet sexual ideals and sexual communal strength on sexual and relationship quality. The findings were largely consistent for both men and women across studies, although some findings were moderated by gender. We report the details of all gender moderations in the Supplemental Materials

\section{Discussion}

The results of Study 1 demonstrate that unmet sexual ideals are associated with lower sexual and relationship quality in romantic relationships. When people report unmet sexual 
ideals in their relationship, both they and their partner reported lower sexual and relationship satisfaction and less commitment to their relationship. Furthermore, people who had partners who were higher in sexual communal strength were buffered against the negative associations between unmet sexual ideals and sexual and relationship quality. Although a person's own sexual communal strength only buffered people against reporting lower commitment in the face of unmet sexual ideals, having a sexually communal partner buffered people against lower sexual satisfaction, relationship satisfaction, and commitment when they reported greater unmet sexual ideals. These findings suggest that having a partner who is highly motivated to meet one's sexual needs (e.g., high in sexual communal strength) may be a more important factor for mitigating unmet sexual ideals than a person's own sexual communal strength. Finally, these results were largely consistent for both men and women, indicating they are largely generalizable across genders (see Supplemental Materials).

\section{Study 2}

Past research has shown relationship ideals can change over time (Charlot, Balzarini, \& Campbell, 2019), and in fact, other related constructs — such as sexual desire (Day et al., 2015) and sexual beliefs (Maxwell et al., 2017) — have been shown to fluctuate day-to-day. Therefore, we expected that people might feel that their sexual ideals are being met more on some days compared to others. In Study 2, we examined reports of unmet sexual ideals in daily life, and whether daily fluctuations in unmet sexual ideals would be associated with subsequent fluctuations in daily sexual satisfaction and relationship quality. To do so, we assessed couples' reports of sexual communal strength at baseline, and their daily perceptions of unmet sexual ideals and sexual and relationship quality over a period of 21 days. Consistent with Study 1, we expected that on days when people reported greater unmet sexual ideals (compared to their own 
average across the study), they would report lower daily sexual satisfaction and relationship quality. Yet, we also expected that people who were high in sexual communal strength, or who had partners who were high in sexual communal strength, would be buffered against lower daily sexual satisfaction and relationship quality when faced with unmet sexual ideals. These hypotheses were pre-registered on the OSF. In addition, we sought to explore a mechanism for why people with partners higher in sexual communal strength are buffered against lower sexual and relationship quality associated with unmet sexual ideals. That is, we tested whether perceiving a partner as highly responsive accounted for the buffering effect of having a partner high in sexual communal strength. In this study, we also explored whether gender influenced the effects and sought to rule out an alternative explanation through assessing whether sexual frequency accounted for the effects of unmet sexual ideals on sexual and relationship quality and the buffering effects of sexual communal strength.

\section{Method}

\section{Participants and Procedure}

We recruited 139 mixed-sex couples from the United States and Canada through advertisements posted on Kijiji and Facebook, an email list of couples who had previously participated in research in our lab, and flyers posted locally. The same eligibility criteria for Study 1 were used in Study 2, except couples in Study 2 had to be cohabitating and had to complete at least three days of the daily diary entries over the course of the study. We aimed to recruit at least 100 couples based on recommendations for achieving sufficient power with dyadic data by Kenny and colleagues (2006). Data from 15 couples were excluded because one or both partners did not consent to participate in the study $(n=5)$ or did not meet inclusion criteria $(n=10)$. Our final sample consisted of 124 couples $(51.2 \%$ female). Post-hoc power 
analyses using an online application for calculating power curves for multi-level studies (Kleiman, 2019) indicated that with 248 participants and 4439 days, we had $99 \%$ power to detect a small effect. Participants were primarily married (44.1\%), Caucasian (72.9\%), and heterosexual $(88.6 \%)$. On average, most participants were in their early 30s $(M=30.70$ years, $S D=8.96)$ and were in long-term relationships $(M=6.73$ years, $S D=5.84)$. See Table 1 for more information about the demographics.

Eligible participants completed a 30-minute background survey, in addition to a brief (10 minutes or less) daily survey every day for 21 consecutive days, completed entirely online and independently from their partner. To maximize participant compliance with the daily diary responses, reminder emails were sent to the participants who had not completed their diaries within 3 hours of their start time each day. On average, participants completed 18.81 diaries across the 21-day study. Participants could earn up to \$35 (CAD) per person for their participation. For more information about the study's measures, please view the measures document on the OSF.

\section{Measures}

In addition to the key variables, both partners reported their sexual frequency and sexual desire (see Table 4 for correlations). For the daily (within-person) measures, we used truncated versions of the focal measures to reduce fatigue, increase efficiency, and minimize participant attrition (Bolger, Davis, \& Rafaeli, 2003).

\section{Baseline Measures.}

Sexual Communal Strength. The Sexual Communal Strength Scale (six items; Muise et al., 2013) was used to assess the tendency to be attuned to and motivated to meet a partner's sexual needs at baseline. Participants completed the measure by rating items such as, "How far 
would you be willing to go to meet your partner's sexual needs?" on a 5-point Likert scale $(1=$ strongly disagree, 5 = strongly agree). Items were reverse coded when necessary and mean aggregated, with higher scores indicating higher sexual communal strength $(\alpha=.68 ; M=4.04$, $S D=0.59)$.

Sexual Frequency. Sexual frequency during the past two weeks was assessed with one item asking participants to estimate the number of times they had engaged in sexual intercourse with their partner (e.g., "Please indicate how many times in the past 2 weeks you and your partner have engaged in sexual activity"). Responses ranged from 0 to 28 times $(M=3.90, S D=$ $3.53)$.

\section{Daily Measures.}

Extent Partner Meets Sexual Ideals. Participants were asked to rate the extent their partner met their sexual ideals each day (a single item; e.g., "To what extent do you feel your partner met your sexual ideals today?"). Possible responses were on a 7-point Likert scale ( $1=$ not all, $7=$ completely) and responses were reverse coded, with higher scores indicating greater unmet sexual ideals $(M=3.07, S D=1.95)$.

Sexual Satisfaction. The GMSEX (three items; Lawrance \& Byers, 1998) asked participants to indicate on 7-point bipolar scales which best described their sexual satisfaction that day (unsatisfying-satisfying, unpleasant-pleasant, and good-bad). Within-person reliability of the items (indicated by $R_{c}$; Bolger \& Laurenceau, 2013) was .96. Items were mean aggregated, with higher scores indicating higher sexual satisfaction $(M=4.79, S D=1.95)$.

Relationship Satisfaction. Participants responded to one item from Hendrick’s (1988) Relationship Assessment Scale regarding how satisfied they were with their relationship that day (a single item; "How satisfied are you with your relationship?"). Possible responses were on a 5- 
point Likert scale $(1=$ not at all, $5=$ a great deal $)$. Higher scores indicate higher relationship satisfaction $(M=4.34, S D=0.83)$.

Commitment. The IMS (three items; Rusbult et al., 1998) was used to assess commitment (e.g., "I am committed to maintaining my relationship with my partner"; $\mathrm{R}_{\mathrm{c}}=.90$ ) among couples. Possible responses were on a 9-point Likert scale $(1=$ do not agree at all, $7=$ agree completely), and the items were mean aggregated, with higher scores indicating higher commitment $(M=6.48, S D=0.95)$.

Perceived Partner Responsiveness. The Perceived Partner Responsiveness Scale (Reis, 2003) was used to assess daily perceptions of a partner's general responsiveness. Participants were asked to rate their perception of their partners responsiveness (three items; e.g., "My partner understands me"; $\left.\mathrm{R}_{\mathrm{c}}=.81\right)$ on a 5-point Likert scale $(1=$ strongly disagree, $5=$ strongly agree). Items were mean aggregated, with higher scores indicating greater perceived partner responsiveness $(M=4.32, S D=0.80)$.

\section{Results}

\section{Unmet Sexual Ideals and Sexual and Relationship Quality in Daily Life}

To test whether unmet sexual ideals are associated with lower relationship and sexual quality in daily life, we tested a two-level crossed model with random intercepts, where persons were crossed with days and nested within couples to account for the fact that both partners completed the daily surveys on the same days (Kenny et al., 2006). The analyses were guided by the APIM, such that both a person's own score and their partner's score for unmet sexual ideals were entered simultaneously as predictors in the model (Kenny et al., 2006). Daily predictors (i.e., unmet sexual ideals) were partitioned into their within- and between-variance components, which were person-mean centered and grand-mean centered respectively (Raudenbush, Bryk, 
Cheong, \& Congdon, 2004; Zhang, Zyphur, \& Preacher, 2009). The benefit of assessing unmet sexual ideals at the daily level is that we can test the effects of within-person differences (i.e., daily fluctuations in unmet sexual ideals) while accounting for between-person differences.

Results from the multi-level dyadic models showed that on days when people reported higher unmet sexual ideals than their average, they felt less satisfied with their sexual $(b=-.64$, $t(4044)=-52.99, p<.001,95 \%$ CI $[-.66,-.61])$ and romantic relationship $(b=-.08, t(3960)=-$ $15.79, p<.001,95 \%$ CI [-.09, -.07]), and also reported feeling less committed to maintaining their relationship $(b=-.06, t(3888)=-8.88, p<.001,95 \%$ CI $[-.07,-.04])$. On days when participants reported having more unmet sexual ideals than typical, their partner also reported lower sexual satisfaction $(b=-.14, t(4045)=-11.85, p<.001,95 \%$ CI $[-.17,-.12])$, relationship satisfaction $(b=-.02, t(3961)=-3.23, p=.001,95 \%$ CI $[-.03,-.01])$, and commitment $(b=-.01$, $t(3889)=-2.06, p=.04,95 \% \mathrm{CI}[-.02,-.001])$.

\section{The Moderating Role of Sexual Communal Strength}

We then tested whether participant's own and their partner's sexual communal strength moderated the daily effects. The analyses were APIM cross-level moderations, such that both a person's own score and their partner's scores for daily unmet sexual ideals (both person mean centered daily predictors and aggregates of daily predictors) and baseline actor sexual communal strength and partner sexual communal strength (grand mean centered), plus the interactions between all terms, were entered simultaneously as predictors in the model (Garcia et al., 2015). We predicted that the association between one's unmet sexual ideals and sexual and relationship quality would be buffered by their own or their partner's sexual communal strength, but we had no expectations about the interaction of the partner's effects and thus these findings can be found in the Supplemental Materials. When an interaction was significant, we proceeded to test simple 
effects at high (one standard deviation above) and low (one standard deviation below) levels of sexual communal strength (Aiken et al., 1991). The analytic plan and the data and syntax for these analyses can be found on the OSF. Correlations between all variables are in Table 4.

Table 4

Correlations Among Key Variables for Study 2

\begin{tabular}{|c|c|c|c|c|c|c|c|}
\hline & 1 & 2 & 3 & 4 & 5 & 6 & 7 \\
\hline 1. Rel. Satisfaction & $.55 * * *$ & & & & & & \\
\hline 2. Sexual Satisfaction & $.45^{* * *}$ & $.48 * * *$ & & & & & \\
\hline 3. Commitment & $.53 * * *$ & $.29 * * *$ & $.38 * * *$ & & & & \\
\hline 4. SCS & $.27 * * *$ & .14 & $.23 *$ & .01 & & & \\
\hline 5. Sexual Frequency & $.18 * * *$ & $.32 * * *$ & .02 & $.17 * * *$ & $.72 * * *$ & & \\
\hline 6. Unmet Sexual Ideals & $-.47 * * *$ & $-.61 * *$ & $-.21 * *$ & -.05 & $-.17 * * *$ & $.42 * * *$ & \\
\hline 7. PPR & $.46 * * *$ & $.66 * * *$ & $.42 * * *$ & $.17^{*}$ & $.24 * * *$ & $-.62 * * *$ & $.56 * * *$ \\
\hline
\end{tabular}

Note: $* * * p<.001, * * p<.01, * p<.05$. Correlations between partners are bolded on the diagonal, with actor's correlations below the diagonal. Baseline reports of specific variables (sexual communal strength and sexual frequency) were included in correlations. All other variables were at the daily level. Daily variables were aggregates across the diary. Rel satisfaction = relationship satisfaction; SCS = sexual communal strength; PPR = perceived partner responsive. The correlation between an individual's unmet sexual ideals and their partner's sexual communal strength was, $r=-.20, p<.05$.

As predicted and consistent with Study 1, the association between daily unmet sexual ideals and a person's own sexual and relationship quality was moderated by their partner's sexual communal strength (see Table 5, Figures 2). That is, people with partners low in sexual 
communal strength reported lower relationship and sexual quality when they reported more unmet ideals (sexual satisfaction: $b=-.67, t(3003)=-32.22, p=.04,95 \%$ CI $[-.71,-.62]$; relationship satisfaction: $b=-.10, t(2993)=-12.02, p<.001,95 \%$ CI [-.12, -.09$]$; and commitment: $b=-.08, t(2988)=-7.53, p<.001,95 \% \mathrm{CI}[-.10,-.06])$. However, the association between daily unmet sexual ideals and sexual and relationship quality were attenuated, although still significant, among individuals who had partners who were higher in sexual communal strength (sexual satisfaction: $b=-.61, t(3001)=-31.73, p<.001,95 \%$ CI $[-.64,-.57]$; relationship satisfaction: $b=-.06, t(2986)=-7.76, p<.001,95 \%$ CI $[-.08,-.05]$; and commitment: $b=-.05, t(2981)=-4.86, p<.001,95 \%$ CI $[-.07,-.03])$.

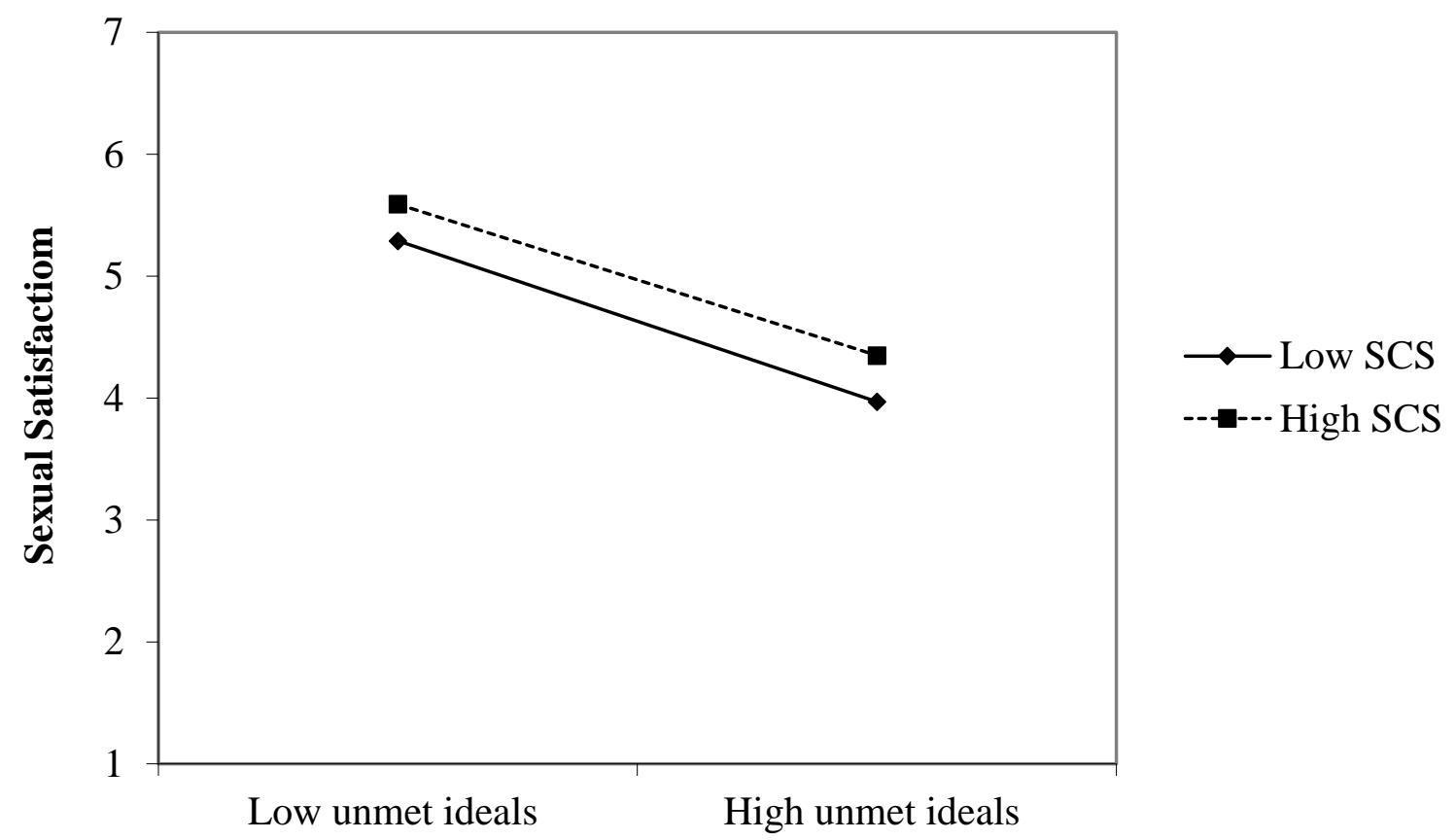

Fig. 2A. The interactive effect of actor's daily unmet sexual ideals and partner's sexual communal strength (SCS) on actor's sexual satisfaction (Study 2). 


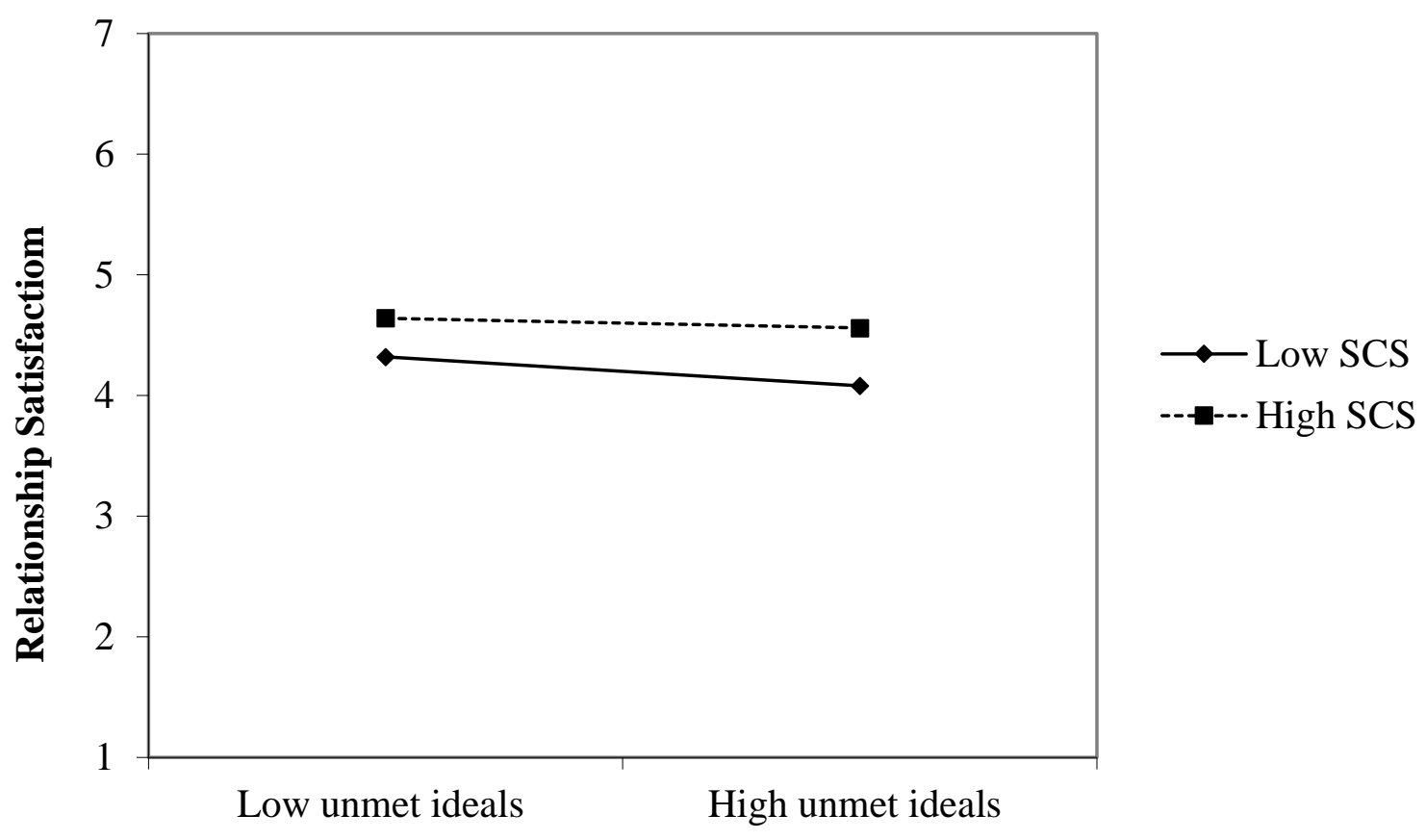

Fig. 2B. The interactive effect of actor's daily unmet sexual ideals and partner's sexual communal strength (SCS) on actor's relationship satisfaction (Study 2).

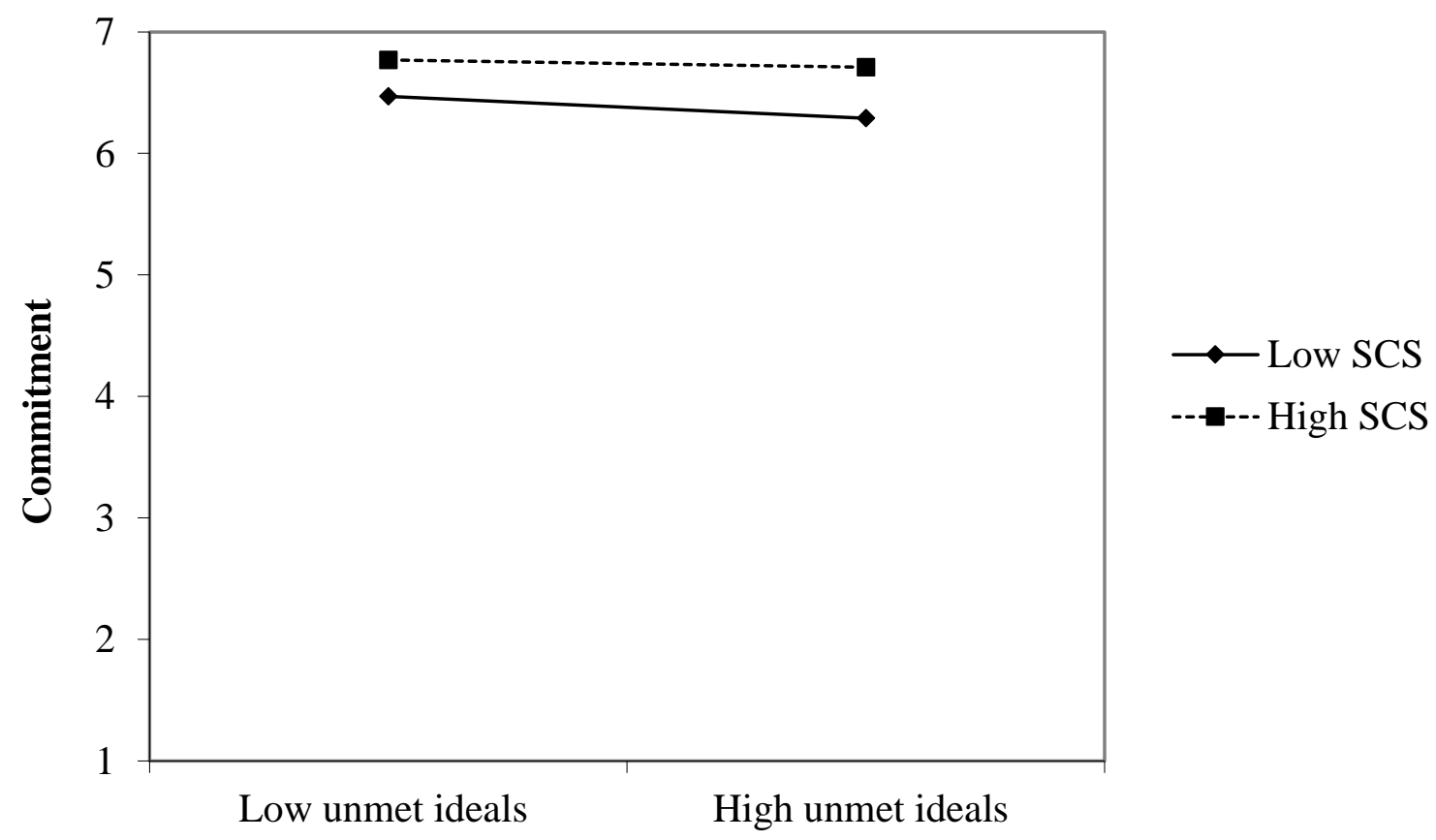


Fig. 2C. The interactive effect of actor's daily unmet sexual ideals and partner's sexual communal strength (SCS) on actor's commitment (Study 2).

Similar to Study 1, the moderating role of one's own sexual communal strength was less consistent across outcomes. While the association between unmet sexual ideals and a person's relationship satisfaction was moderated by their own sexual communal strength (see Table 6) such that the association between daily unmet sexual ideals and relationship satisfaction was attenuated, although still significant among individuals who were higher in sexual communal strength $(b=-.07, t(2984)=-9.06, p<.001,95 \%$ CI $[-.09,-.06])$, compared to individuals who were lower in sexual communal strength $(b=-.09, t(2993)=-10.90, p<.001,95 \%$ CI $[-.11$, .08]) — the effects for sexual satisfaction and commitment were mixed. The association between unmet sexual ideals and a person's own sexual satisfaction was moderated by their own sexual communal strength; however, the effect was in the opposite direction to our predictions. More specifically, unmet sexual ideals were associated with lower sexual satisfaction for individuals who were high in sexual communal strength $(b=-.68, t(2998)=-35.99, p<.001,95 \%$ CI $[-.71$, .64]) and the association was slightly attenuated for people lower in sexual communal strength ( $b$ $=-.59, t(3003)=-28.71, p<.001,95 \%$ CI $[-.64,-.55])$, while the association between unmet sexual ideals and commitment was not moderated by their own sexual communal strength $(p=$ $.119)$.

Table 5

APIM Models with Daily Reports of Unmet Sexual Ideals (USI) and Baseline Sexual Communal Strength (SCS) Predicting Daily Sexual and Relationship Quality in Study 2

Relationship

Satisfaction
Sexual

Satisfaction
Commitment 


\begin{tabular}{cccccc}
\hline$b$ & $S E$ & $b$ & $S E$ & $b$ & $S E$
\end{tabular}

\begin{tabular}{lcccccc}
\hline Fixed Effects & & & & & & \\
Intercept & 4.40 & $.05^{* * *}$ & 4.80 & $.07 * * *$ & 6.56 & $.06^{* * *}$ \\
Actor (A) USI & -.08 & $.01 * * *$ & -.64 & $.01 * * *$ & -.06 & $.01 * * *$ \\
Partner (P) USI & -.02 & $.01 * * *$ & -.13 & $.01 * * *$ & -.01 & .01 \\
A SCS & .21 & $.07 * *$ & .18 & .10 & .27 & $.09 * *$ \\
P SCS & .20 & $.07 * *$ & .17 & .10 & .18 & $.09 *$ \\
A USI x A SCS & .02 & $.01 *$ & -.07 & $.02 * *$ & .02 & .01 \\
A USI x P SCS & .04 & $.01 * * *$ & .05 & $.02 *$ & .03 & $.01 *$ \\
P USI x A SCS & .02 & $.01 *$ & .05 & $.02 *$ & .02 & .01 \\
P USI x P SCS & .02 & .01 & -.06 & $.02 * *$ & .01 & .01 \\
\hline
\end{tabular}

Note: $* * * p<.001, * * p<.01, * p<.05$. Note that the main effect coefficients differ slightly from those reported in-text values because the in-text values are from the main effects only models and the values in the table are from the full model with interactions included.

\section{The Mediating Role of Perceived Partner Responsiveness (exploratory)}

Next, we explored whether one reason why people with partners higher in sexual communal strength are buffered against feeling lower levels of sexual and relationship quality when they have unmet sexual ideals is because they perceive their partners to be highly responsive. We conducted a mediated moderation following the guidelines by Muller and colleagues (2005) and using the Monte Carlo Method for Assessing Mediation (MCMAM; Selig $\&$ Preacher, 2008) to test the significance of the indirect effects (using 95\% Confidence Intervals) of the moderation between unmet sexual ideals and a partner's sexual communal 
strength on sexual and relationship quality through perceived partner responsiveness. See Figure 3A-3C for the models tested.

First, we tested whether the association between unmet sexual ideals and perceived partner responsiveness (the mediator) was moderated by partner sexual communal strength. In fact, this was the case, $b=.04, t(2921)=5.04, p<.001$ (see Figure 3A-3C). People with partners low in sexual communal strength reported that unmet sexual ideals were associated with lower perceived partner responsiveness, $b=-.10, t(3003)=-12.96, p<.001$, whereas this effects was attenuated for people with partner's high in sexual communal strength, $b=-.05, t(3004)=-6.58$, $p<.001$. Next, we tested whether perceived partner responsiveness accounted for the moderated association between unmet sexual ideals and each outcome by partner sexual communal strength. We found a significant indirect effect for relationship satisfaction (95\% CI [.01, .03]), sexual satisfaction (95\% CI [.01, .03]), and commitment (95\% CI [.01, .03]), and when perceived partner responsiveness was entered into the model, the moderated association between unmet sexual ideals and relationship quality by partner sexual communal strength became nonsignificant. This pattern of results is consistent with mediated moderation and suggests that one reason why people with partners who are higher in sexual communal strength are buffered against experiencing lower daily sexual and relationship quality in response to having unmet sexual ideals is in part because they perceive their partner as more responsive to their needs. 


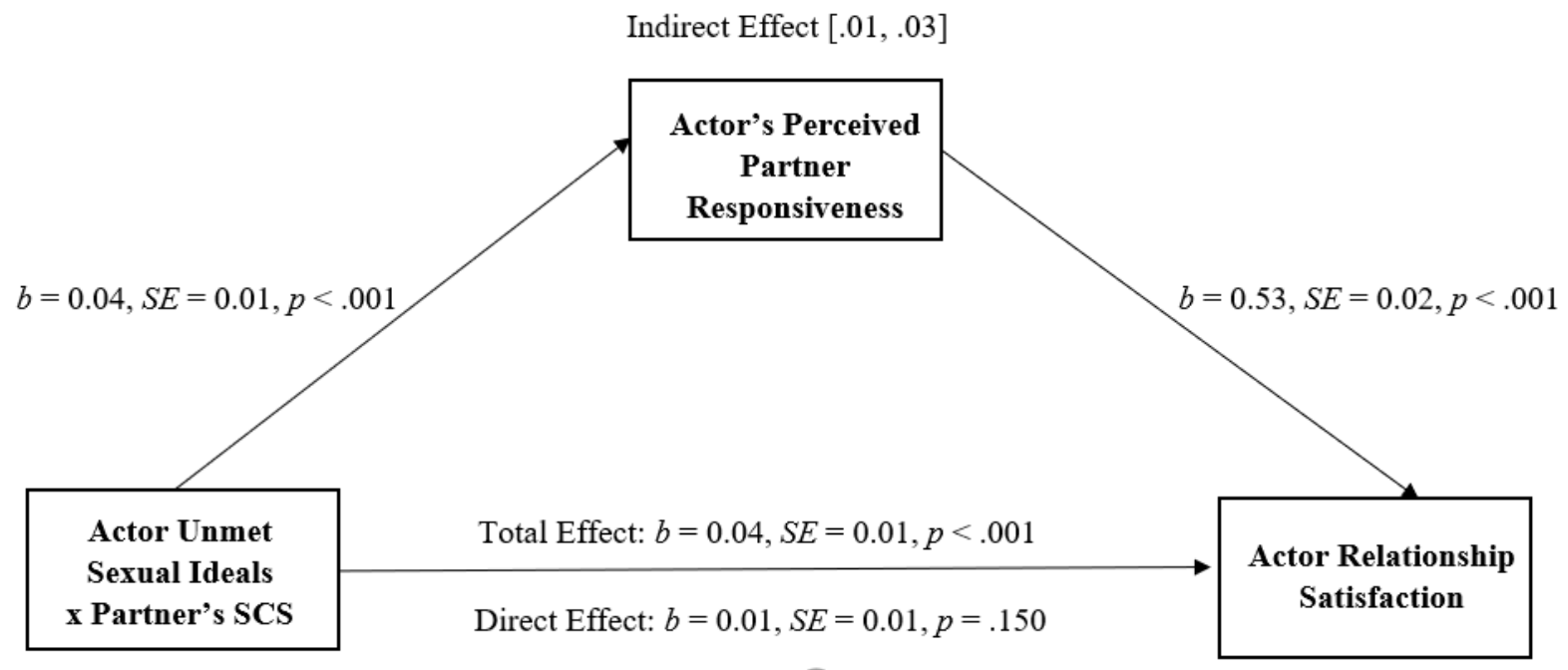

Fig. 3A. Actor's perceived partner responsiveness as a mediator of the interactive effect of actor's unmet sexual ideals and partner's sexual communal strength (SCS) on actor's relationship satisfaction.

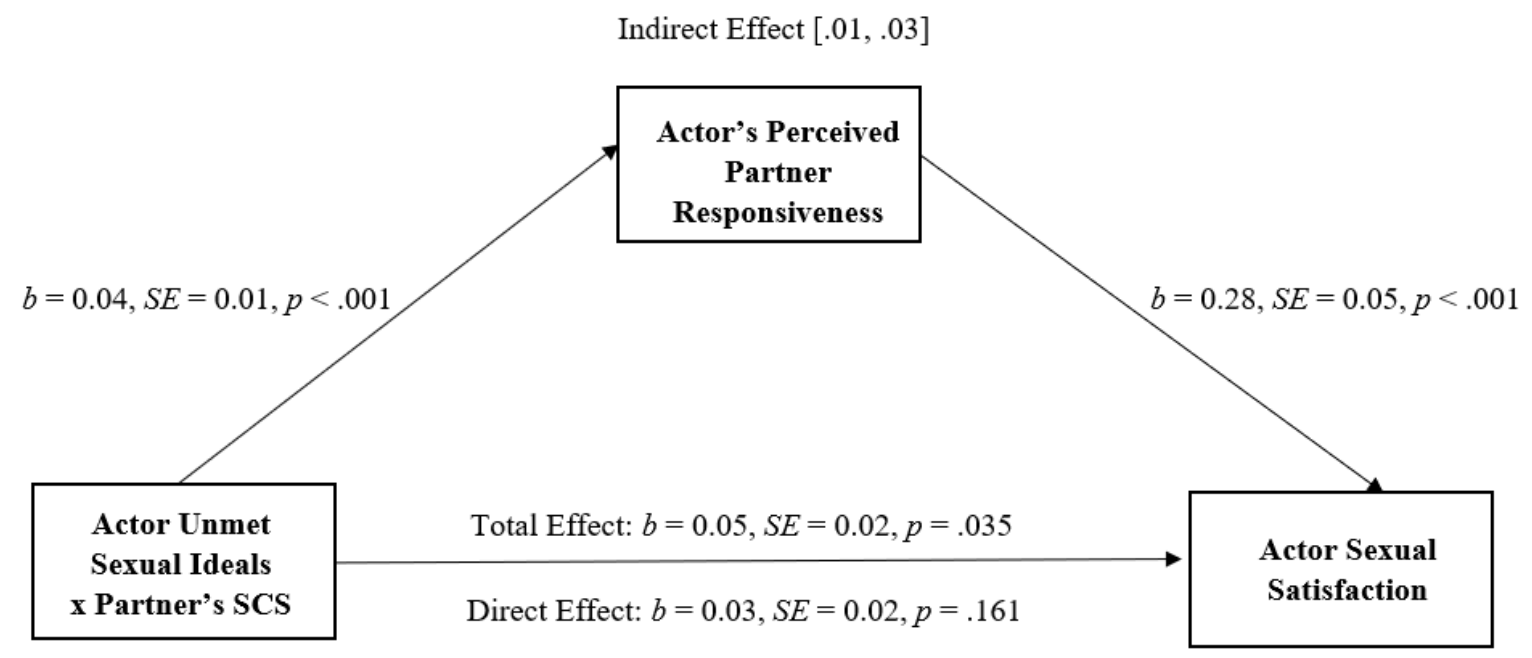

Fig. 3B. Actor's perceived partner responsiveness as a mediator of the interactive effect of actor's unmet sexual ideals and partner's sexual communal strength (SCS) on actor's sexual satisfaction. 


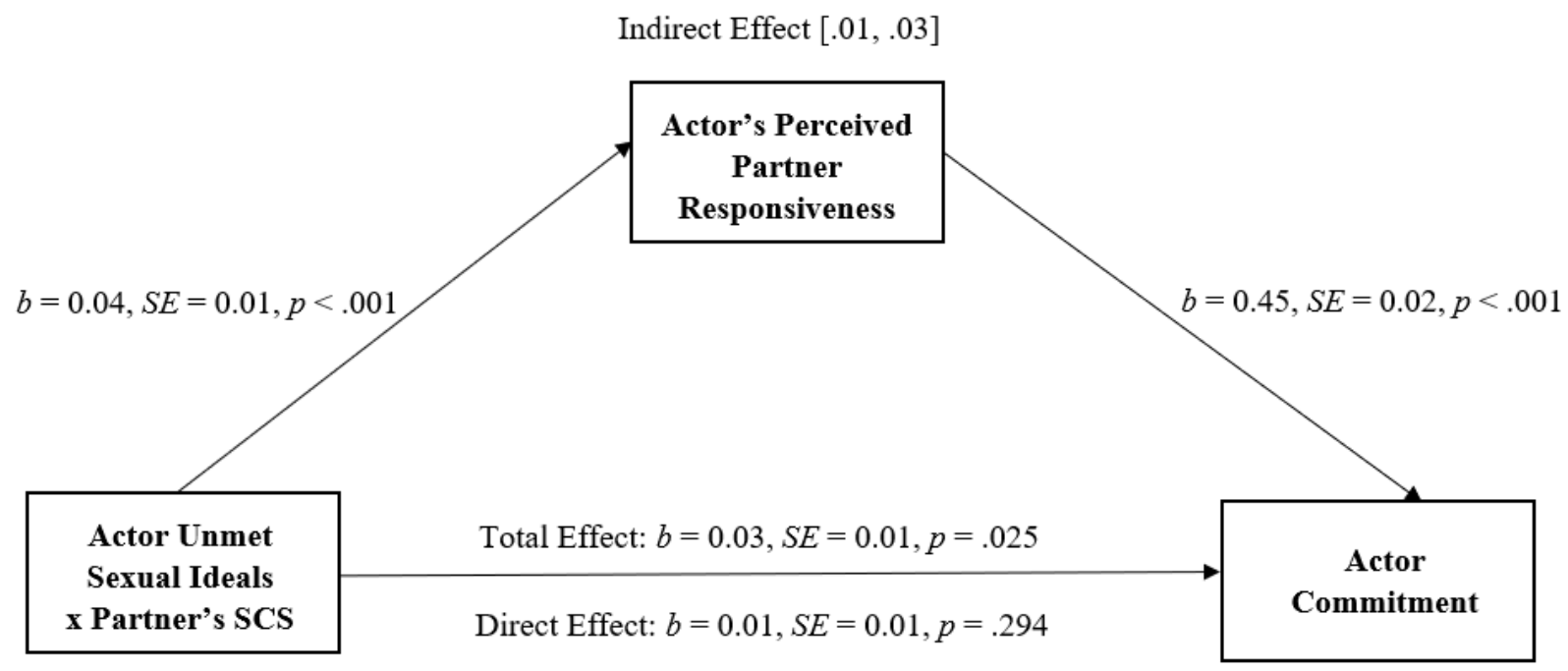

Fig. 3C. Actor's perceived partner responsiveness as a mediator of the interactive effect of actor's unmet sexual ideals and partner's sexual communal strength (SCS) on actor's commitment.

\section{Ruling Out Alternative Explanations and Providing Evidence for Generalizability of the Findings.}

Given that the data are correlational, in exploratory analyses we aimed to isolate the direction of the effects by accounting for the previous day's outcome variable. Accounting for people's satisfaction on the previous day, on days when they reported greater unmet ideals than they did on average they reported lower sexual $(b=-.64, t(3814)=-51.50, p<.001,95 \%$ CI $[-$ $.66,-.61])$ and relationship satisfaction $(b=-.08, t(3730)=-14.94, p<.001,95 \%$ CI $[-.09,-.07])$, and poorer commitment $(b=-.05, t(3721)=-8.05, p<.001,95 \% \mathrm{CI}[-.06,-.04])$, and their partner's reported less sexual $(b=-.15, t(3812)=-12.01, p<.001,95 \%$ CI $[-.17,-.12])$ and relationship satisfaction $(b=-.02, t(3728)=-3.29, p=.001,95 \%$ CI $[-.03,-.01])$, and marginally lower commitment $(b=-.01, t(3722)=-1.95, p=.051,95 \%$ CI $[-.02, .00]) .^{3}$

\footnotetext{
${ }^{3}$ The effects were constrained to the same day, null effects emerged for next day lagged effects.
} 
Additionally, when controlling for the previous days outcome, the association between daily unmet sexual ideals and a person's own sexual and relationship satisfaction was still moderated by their partner's sexual communal strength, though the effect for commitment was reduced to marginal (see Table 6). That is, people with partners low in sexual communal strength reported lower sexual and relationship satisfaction when they reported more unmet ideals (sexual satisfaction: $b=-.67, t(2853)=-31.66, p<.001,95 \%$ CI $[-.71,-.63]$; and relationship satisfaction: $b=-.11, t(2839)=-11.87, p<.001,95 \%$ CI $[-.12,-.09])$ controlling for the relevant outcomes on the previous day. The association between daily unmet sexual ideals and sexual and relationship quality were attenuated, although still significant, among individuals who had partners who were higher in sexual communal strength (sexual satisfaction: $b=-.61, t(2847)=-$ 31.60, $p<.001,95 \%$ CI [-.65, -.57]; and relationship satisfaction: $b=-.06, t(2834)=-7.16, p<$ $.001,95 \%$ CI $[-.07,-.04])$ controlling for the outcomes on the previous day.

Moreover, the association between unmet sexual ideals and a person's sexual and relationship satisfaction was moderated by their own sexual communal strength when controlling for outcomes on the previous day (see Table 6) — but the results were in divergent directions. More specifically, the association between daily unmet sexual ideals and relationship satisfaction was attenuated, although still significant among individuals who were higher in sexual communal strength $(b=-.07, t(2832)=-8.60, p<.001,95 \%$ CI $[-.08,-.05])$, compared to individuals who were lower in sexual communal strength $(b=-.10, t(2838)=-10.66, p<.001$, 95\% CI [-.11, -.08]). In contrast, the association between daily unmet sexual ideals and relationship satisfaction was larger among individuals who were higher in sexual communal strength $(b=-.68, t(2845)=-35.55, p<.001,95 \%$ CI $[-.71,-.64])$, compared to individuals who were lower in sexual communal strength $(b=-.60, t(2852)=-28.48, p<.001,95 \%$ CI $[-.65,-$ 
.56]). The association between unmet sexual ideals and commitment was not moderated by their own sexual communal strength $(p=.263)$. More information about partner interactions can be found in the Supplement Materials.

Table 6

APIM Models with Daily Unmet Sexual Ideals (USI) and Baseline Sexual Communal Strength (SCS) Predicting Daily Sexual and Relationship Quality Controlling for the Previous Days Outcome in Study 2

\section{Relationship Commitment}

Satisfaction

Satisfaction

\begin{tabular}{llllll}
\hline $\boldsymbol{B}$ & $S E$ & $B$ & $S E$ & $b$ & $S E$
\end{tabular}

\section{Fixed Effects}

Intercept

4.41

$.05 * * *$

4.80

$.07 * * *$

6.57

$.06 * * *$

Actor (A) USI

$-.08 \quad .01 * * *$

$-.64$

$.01 * * *$

$-.06$

$.01 * * *$

Partner (P) USI

$$
-.02
$$

$.01 * * *$

$-.14$

$.01 * * *$

$-.01$

.01

A SCS

.20

$.07 *$

.19

.10

.27

$.09 *$

P SCS

.21

$.07 *$

.18

.10

.18

$.09 *$

Outcome Yesterday

.12

$.02 * * *$

.04

$.01 *$

.17

$.02 * * *$

A USIs x A SCS

.02

$.01 *$

$-.06$

$.02 *$

.01

.01

A USI x P SCS

.04

$.01 * * *$

.05

$.02 *$

.02

.01

P USI x A SCS

.02

$.01 *$

.05

$.02 *$

.02

.01

P USI x P SCS

.02

.01

$-.07$

$.02 *$

.01

.01 
Note: $* * * p<.001,{ }^{* *} p<.01,{ }^{*} p<.05$. Note that the main effect coefficients differ slightly from those reported in-text values because the in-text values are from the main effects only models and the values in the table are from the full model with interactions included.

Next, we conducted additional exploratory analyses to rule out possible alternative explanations and provide evidence for the generalizability of our findings. First, our primary prediction is that unmet sexual ideals are associated with lower sexual and relationship quality, but people who are higher or have partners who are higher in sexual communal strength are buffered against the negative association between unmet sexual ideals and sexual and relationship quality. Given that unmet ideals are associated with having less frequent sex $(r=-$ $.17, p=.013$ ), and sexual communal strength is associated with more frequent sex (actor sexual communal strength: $r=.17, p=.025$; partner sexual communal strength; $r=.19, p=.014$ ), we wanted to rule out the possibility that the buffering effect of sexual communal strength is solely attributed to engaging in more frequent sex to increase our confidence that it is sexual communal strength buffering the effects and not increased sexual activity. To test this, we re-ran the daily analyses controlling for couples' sexual frequency at baseline, and all of the significant effects reported above remained significant.

\section{Discussion}

This study provides support for the negative effects of unmet sexual ideals and the moderating role of a partner's sexual communal strength on associations between unmet sexual ideals and sexual and relationship quality in daily life. The buffering effects of a partner's sexual communal strength were robust and emerged even when controlling for couples' sexual frequency and were largely consistent across men and women (with the exception of the results for commitment). However, as in Study 1, evidence for the moderating role of one's own sexual 
communal strength were less consistent, presenting null results or contrasting findings across the outcomes. Having a partner who was high in sexual communal strength had a more consistent buffering effect on the associations between unmet sexual ideals and lower relationship quality than one's own sexual communal strength. Notably, even when the effects were moderated, the negative effects of unmet ideals were still significant for people with highly communal partners. That is, at least in daily life, even if a partner is sexually responsive, unmet sexual ideals are still associated with lower sexual satisfaction and relationship quality. This study also provides initial insight into a potential mechanism for the buffering effect of a partner's sexual communal strength on the association between unmet sexual ideals and sexual and relationship quality. Exploratory analyses suggest that people with partners who are higher in sexual communal strength are buffered against experiencing lower daily sexual and relationship quality in response to having unmet sexual ideals in part because they perceive their partner as more responsive to their needs.

\section{Study 3}

Having demonstrated the effects of unmet sexual ideals on sexual and relationship quality in dyadic cross-sectional (Study 1) and daily experience (Study 2) studies, we next sought to replicate and extend the findings by examining whether unmet sexual ideals and the moderation by sexual communal strength are associated with changes in sexual and relationship quality over time. To do so, we assessed couples' reports of sexual communal strength at baseline, their perceptions of unmet sexual ideals and sexual and relationship quality in daily life over a period of 21 days, and their sexual and relationship quality three months later. Consistent with Study 2, we expected that on days when people reported greater unmet sexual ideals (compared to their own average), they would report lower daily sexual and relationship satisfaction, and 
commitment. We further predicted that when individuals reported more unmet sexual ideals over the 21-day diary study (aggregated across daily measures), both partners would report feeling less satisfied with their sexual and romantic relationship and lower commitment three months later (controlling for their baseline reports). However, we expected that people who had partners who were high in sexual communal strength would be buffered against lower daily sexual satisfaction and relationship quality when faced with unmet sexual ideals and would not report lower sexual satisfaction and relationship quality three months later. Because the moderating role of one's own sexual communal strength in buffering the association between unmet sexual ideals and sexual satisfaction and relationship quality were inconsistent in Studies 1 and 2, we did not have any specific predictions for this study. Overall, based on the findings of our previous studies we expected one's partner's sexual communal strength would be a stronger and more consistent moderator of the negative associations between unmet sexual ideals and sexual and relationship quality. Additionally, in Study 3, we also seek to confirm a mechanism for the buffering effect of partner's sexual communal strength. More specifically, in Study 2 we found support for mediated moderation where the moderation between unmet sexual ideals and partner sexual communal strength on the daily outcomes (sexual satisfaction, relationship satisfaction, commitment) was mediated by daily perceived partner responsiveness. These analyses suggest that people with partners higher in sexual communal strength were buffered against experiencing lower daily sexual and relationship quality in response to having unmet sexual ideals in part because they perceive their partner as more responsive to their needs. In the current research, we seek to replicate and extend the previous study by examining both perceived partner responsiveness generally, and perceived partner responsiveness specific to sexuality as 
mechanisms for the buffering effect of partner sexual communal strength. These hypotheses were pre-registered on the OSF.

\section{Method}

\section{Participants and Procedure}

A new sample of mixed-sex couples $(N=126)$ were recruited from various sources in Canada and the United States, including Kijiji, Facebook, Reddit, Craigslist, an email list of past participants interested in participating in future research, and advertisements (e.g., Canadian university campuses, public transportation centers). The same eligibility criteria for Studies 2 and 3 were used in Study 3, except couples in Study 3 had to be sexually active in their current relationship, had to be living together or seeing each other $5 / 7$ days per week, and in a relationship for two or more years. One couple was excluded because they only completed the baseline survey of the study $(n=2)$. Post-hoc power analyses for calculating power curves for multi-level studies (Kleiman, 2019) indicated that with 252 participants and 4488 days, we had $99 \%$ power to detect a small effect. Most participants were white $(65.6 \%)$ heterosexuals $(81.4 \%)$ who were on average in their early $30 \mathrm{~s}(M=32.63$ years, $S D=10.17)$ and were in long-term relationships $(M=5.11$ years, $S D=3.51)$. The sample consisted of men $(n=115)$, women $(n=$ 124), and two participants who identified with another gender (See Table 1 for more information about the demographics).

The procedure of Study 3 was the same as Study 2, except after completing the daily surveys, couples were asked to take a follow-up questionnaire that included measures of sexual and relationship quality three months later. On average, participants completed 18.39 diaries across the 21-day study, and 214 participants (88\%) completed the follow-up survey. As compensation for taking part in this study, couples were provided the opportunity to earn up to 
\$120 CAD/\$96 USD (\$60 CAD/ \$48 USD for each couple member) if they participated in all aspects of the study. For more information about the study's procedures and measures, please view the measures document on the OSF.

\section{Baseline and Follow-up Measures}

Baseline measures were assessed the day before the 21-day diary study began. For the follow-up survey, couples were asked to complete the measures three months after completing the 21-day diary study.

Sexual Communal Strength. The Sexual Communal Strength Scale (four items; Muise et al., 2013) was used to assess the tendency to be attuned to and motivated to meet a partner's sexual needs. Participants rated items ( "How far would you be willing to go to meet your partner's sexual needs?") on a 5-point Likert scale ( $0=$ not at all, $4=$ extremely). Items were mean aggregated, with higher scores indicating more sexual communal strength $(\alpha=.74 ; M=$ $3.33, S D=0.58){ }^{4}$

Sexual Satisfaction. The GMSEX (Lawrance \& Byers, 1998) asked participants to indicate on 7-point bipolar scales which best describes their overall sexual satisfaction at baseline and three months later (five items; e.g., bad-good, unpleasant-pleasant, negativepositive, unsatisfying-satisfying, worthless-valuable). Items were mean aggregated, with higher scores indicating higher sexual satisfaction at baseline $(\alpha=.96 ; M=6.01, S D=1.40)$ and in the follow-up survey $(\alpha=.97 ; M=5.83, S D=1.50)$.

Relationship Satisfaction. The Perceived Relationship Quality Component (PRQC;

Fletcher, et al., 2000) was used to assess relationship satisfaction at baseline and three months

\footnotetext{
${ }^{4}$ Note that we pre-registered and initially included the standard 6-item measure, but the reliability was low when we included the two reverse-coded items. The measure was reliable, however, without the reverse-coded items. As such, we proceeded with the 4-item measure.
} 
later (three items; e.g., "How satisfied are you with your relationship?; How content are you with your relationship?; How happy are you with your relationship?"). Possible responses were on a 7-point scale $(1=$ not at all, 7 = extremely), and items were mean aggregated, with higher scores indicating higher relationship satisfaction at baseline $(\alpha=.95 ; M=6.14, S D=0.92)$ and in the follow-up survey $(\alpha=.94 ; M=5.97, S D=1.16)$.

Commitment. The PRQC (Fletcher, et al., 2000) was used to assess commitment at baseline (two items; e.g., "How committed are you to your relationship?"; "How dedicated are you to your relationship?") and three months later (a single item: e.g., "How committed are you to your relationship?"). Possible responses were on a 7 -point scale $(1=$ not at all, $7=$ extremely $)$, and items were mean aggregated (for baseline), with higher scores indicating higher commitment at baseline $(\alpha=.83 ; M=6.69, S D=0.59)$ and in the follow-up survey $(M=6.58, S D=0.86)$.

Sexual Frequency. Sexual frequency during the past 30 days was assessed for various types of sexual activity (seven items; e.g., oral sex [giving to partner], oral sex [receiving from partner], giving manual stimulation [touching or massaging your partner's genitals], receiving manual stimulation [your partner touching or massaging your genitals], manual stimulation [masturbation; alone], sexual intercourse with vaginal penetration, sexual intercourse with anal penetration). Possible response options were on a 6 -point scale $(0=$ not at all, $6=$ more than once a day), with higher scores indicating greater sexual frequency $(\alpha=.85, M=1.98, S D$ $=1.03)$.

Sexual Desire. Sexual desire during the past 30 days was assessed with two items (e.g., "Over the past 4 weeks, how often did you feel sexual desire or interest for your partner?" and “Over the past 4 weeks, how would you rate your level (degree) of sexual desire or interest?”). Possible responses were on a 5-point scale $(1=$ almost always or always/very high, $5=$ almost 
never or never/very low or none at all), and the items were reverse coded and mean aggregated with higher scores indicating greater sexual desire $(\alpha=.82, M=3.79, S D=0.90)$.

\section{Daily Measures}

We used shortened versions of the focal measures in the daily portion of the diary study to reduce fatigue, increase efficiency, and minimize participant attrition (Bolger et al., 2003).

Extent Partner Meets Sexual Ideals. Participants were asked to rate the extent their partner met their ideals (a single item; e.g., "My partner met my sexual ideals today”) each day. Possible responses were on a 7 -point scale $(1=$ strongly disagree, $7=$ strongly agree $)$ and responses were reverse coded, with higher scores indicating greater unmet ideals $(M=3.19, S D$ $=1.94)$.

Perceived Partner Responsiveness. Three items were used to assess general responsiveness (modified from Maisel \& Gable, 2009 and Reis, 2003). Participants were asked to rate their perception of their partner's responsiveness (e.g., "Today, I felt understood by my partner") on a 7-point scale $(1=$ not at all, $7=a$ lot $)$. Items were mean aggregated, with higher scores indicating greater perceived partner responsiveness $\left(\mathrm{R}_{\mathrm{c}}=.68 ; M=4.07, S D=1.02\right)$.

Perceived Partner Sexual Responsiveness. A single item from the Partner-Focused Positive Relationship Behaviours Scale (Maxwell, Joel, Peetz, MacDonald, \& Baucom, 2019) was used to assess perceived partner sexual responsiveness. Participants were asked to rate their perception of their partner's sexual responsiveness (e.g., “Today, my partner was perceptive of my sexual needs") on a 7 -point Likert scale $(1=$ not at all, $7=$ very much $)$, with higher scores indicating greater perceived partner sexual responsiveness $(M=4.84, S D=2.03)$.

Sexual Satisfaction. Five items from the GMSEX (Lawrance \& Byers, 1998) asked participants to indicate on 7-point bipolar scales which best describes their sexual satisfaction 
that day (e.g., bad-good, unpleasant-pleasant, negative-positive, unsatisfying-satisfying, worthless-valuable). The items were mean aggregated, with higher scores indicating higher sexual satisfaction $\left(\mathrm{R}_{\mathrm{c}}=.96 ; M=5.55, S D=1.68\right)$.

Relationship Satisfaction. Participants responded to one item from Fletcher et al.'s (2000) PRQC regarding how satisfied they were with their relationship that day (e.g., "How satisfied were you with your relationship?"). Possible responses were on a 7 -point scale $(1=n o t$ at all, $7=$ extremely $)$, with higher scores indicating higher relationship satisfaction $(M=6.04, S D$ $=1.25)$.

Commitment. One item from the PRQC Scale (Fletcher et al., 2000) was used to assess commitment (e.g., "How committed were you to your relationship?”) among couples. Possible responses were on a 7 -point scale $(1=$ not at all, $7=$ extremely $)$, with higher scores indicating higher commitment $(M=6.46, S D=1.00)$.

\section{Results}

\section{Unmet Sexual Ideals and Sexual and Relationship Quality in Daily Life}

The analytic strategy for daily effects were the same as Study 2, and the analytic plan and the data and syntax for these analyses can be found on the OSF. Correlations between primary measures can be found in Table 7.

Table 7

Correlations Among Focal Variables for Study 3

\section{1}

1. Rel Satisfaction

2

3

$.52 * * *$

2. Sexual Satisfaction

$.54 * * * \quad .47 * * *$ 


$\begin{array}{lrrrrrrrr}\text { 3. Commitment } & .59^{* * *} & .32^{* * *} & .13^{*} & & & & \\ \text { 4. SCS } & .06 & .10 & .13^{1} & -.12 * * & & & \\ \text { 5. Sexual Frequency } & .20^{* *} & .30^{* *} & .09 & .14^{*} & . \mathbf{1 3}^{1} & & \\ \text { 6. Sexual Desire } & .17^{* *} & .26^{* * *} & .15^{*} & .45^{* * *} & .37^{* * *} & .06 & \\ \text { 7. Unmet Sexual Ideals } & -.50^{* * *} & -.56^{* * *} & -.32^{* * *} & -.08 & -.31^{* * *} & -.25^{* * *} & . \mathbf{4 4} * * * & \\ \text { 8. PPR } & .78^{* * *} & .53^{* * *} & .50^{* * *} & .12^{*} & .27^{* * *} & .22^{* *} & -.48^{* * *} & \\ \text { 9. PPSR } & .51^{* * *} & .67^{* * *} & .27 * * * & .02 & .31^{* * *} & .27^{* * *} & -59^{* * *} & .51^{* * *}\end{array}$

Note: $* * * p<.001, * * p<.01, * p<.05$. Correlations between partners are bolded on the diagonal, with actor's correlations below the diagonal. Baseline reports of specific variables (sexual communal strength, sexual frequency, and sexual desire) were included in correlations. All other variables were at the daily level. Daily variables were aggregates across the diary. Rel satisfaction $=$ relationship satisfaction; $\mathrm{SCS}=$ sexual communal strength; $\mathrm{PPR}=$ perceived partner responsiveness; PPSR $=$ Perceived Partner Sexual Responsiveness. ${ }^{1}=$ Marginal at $p=$ .05-.06. The correlation between an individual's unmet sexual ideals and their partner's sexual communal strength was, $r=-.25, p<.001$.

Results of the multi-level dyadic models showed that, consistent with Study 2 and our pre-registered predictions, on days when people reported higher unmet sexual ideals (compared to their own average), they reported feeling less satisfied with their sexual $(b=-.27, t(3785)=-$ $26.61, p<.001,95 \% \mathrm{CI}[-.29,-.25])$ and romantic relationship $(b=-.22, t(3925)=-22.19, p<$ $.001,95 \% \mathrm{CI}[-.23,-.19])$, and reported being less committed to their relationship ( $b=-.10$, $t(3709)=-12.76, p<.001,95 \%$ CI $[-.11,-.08])$. Similarly, on days when people reported higher unmet sexual ideals, their partner reported lower sexual satisfaction $(b=-.08, t(3785)=-7.71, p$ 
$<.001,95 \%$ CI $[-.10,-.06])$, relationship satisfaction $(b=-.06, t(3925)=-6.66, p=.001,95 \% \mathrm{CI}$ $[-.08,-.05])$, and commitment $(b=-.02, t(3709)=-2.25, p=.02,95 \%$ CI $[-.03,-.00])$.

\section{Moderating Role of Sexual Communal Strength}

The analytic strategy for examining the moderating role of sexual communal strength in daily effects were the same as Study 2. First, as predicted, the association between a person's daily unmet sexual ideals and their own sexual satisfaction was moderated by their partner's sexual communal strength (see Table 8). That is, people with partners low in sexual communal strength reported significantly lower sexual satisfaction on days when they had higher unmet sexual ideals $(b=-.31, t(3972)=-21.36, p<.001,95 \%$ CI $[-.33,-.28])$, but the association between daily unmet sexual ideals and lower sexual satisfaction was significantly attenuated among people with partners who were higher in sexual communal strength $(b=-.21, t(3847)=-$ $15.29, p<.001,95 \%$ CI $[-.24,-.05])$. Contrary to our predictions and findings from Studies 2 and 3 , the association between unmet sexual ideals and a person's own relationship satisfaction $(p=$ $.286)$ and commitment $(p=.286)$ was not moderated by their partner's sexual communal strength. Additionally, the association between unmet sexual ideals and a person's own sexual satisfaction $(p=.419)$, relationship satisfaction $(p=.610)$ and commitment $(p=.626)$ was not moderated by their own sexual communal strength (see Table 8).

Table 8

APIM Models with Daily Unmet Sexual Ideals (USI) and Baseline Sexual Communal Strength (SCS) Predicting Daily Sexual and Relationship Quality in Study 3

Relationship Commitment

Satisfaction Satisfaction

\begin{tabular}{llllll}
\hline$b$ & $S E$ & $b$ & $S E$ & $b$ & $S E$
\end{tabular}




\section{Fixed Effects}

$\begin{array}{lcccccc}\text { Intercept } & 6.05 & .05 * * * & 5.49 & .09 * * * & 6.46 & .06 * * * \\ \text { Actor (A) USI } & -.21 & .01 * * * & -.25 & .01 * * * & -.10 & .01 * * * \\ \text { Partner (P) USI } & -.07 & .01 * * * & -.09 & .01 * * * & -.01 & .01 \\ \text { A SCS } & .14 & .08 & .00 & .15 & .13 & .09 \\ \text { P SCS } & .07 & .08 & -.05 & .15 & .09 & .09 \\ \text { A USIs x A SCS } & .01 & .02 & -.01 & .02 & .01 & .01 \\ \text { A USI x P SCS } & -.02 & .02 & .08 & .02 * * * & -.01 & .01 \\ \text { P USI x A SCS } & -.00 & .02 & .06 & .02 * * * & -.00 & .01 \\ \text { P USI x P SCS } & .03 & .021 & .03 & .02 & .00 & .01\end{array}$

Note: ${ }^{* * *} p<.001,{ }^{* *} p<.01,{ }^{*} p<.05,{ }^{1}$ indicates $\mathrm{p}$-value is marginal at $p=.05-.06$. Note that the main effect coefficients differ slightly from those reported in-text values because the in-text values are from the main effects only models and the values in the table are from the full model with interactions included.

\section{The Mediating Role of Perceived Partner General and Sexual Responsiveness}

Next, we tested whether perceived partner responsiveness (pre-registered) or perceived partner sexual responsiveness (exploratory) are reasons why people with partners higher in sexual communal strength are buffered against feeling less sexual and relationship quality when they have unmet sexual ideals. First, we tested whether the association between unmet sexual ideals and perceived partner responsiveness (the mediator) was moderated by partner sexual communal strength, and inconsistent with our findings from Study 2 the effect was not significant, although trending $(p=.082)$. Next, we tested whether the association between unmet sexual ideals and perceived partner sexual responsiveness (the mediator) was moderated by 
partner sexual communal strength. In fact, this was the case, $b=.08, t(3821)=3.10, p=.002$. People with partners low in sexual communal strength reported that on days when they reported higher unmet sexual ideals they perceived their partner as significantly less responsive to their sexual needs, $b=-.56, t(3908)=-26.63, p<.001$, whereas the association between unmet sexual ideals and perceived partner sexual responsiveness was attenuated for people with partners high in sexual communal strength, $b=-.47, t(3934)=-23.26, p<.001$. Therefore, given there was not a significant association with perceived partner general responsiveness, we tested perceived partner sexual responsiveness as the mediator. We assessed the indirect effects and found a significant indirect effect for sexual satisfaction (95\% CI $[.01, .03])$, relationship satisfaction $(95 \%$ CI $[.003, .02])$, and commitment $(95 \%$ CI $[.003, .02])$. This pattern of results is consistent with mediated moderation and suggests that people with partners high in sexual communal strength are buffered against experiencing lower daily sexual satisfaction and relationship quality in response to having unmet sexual ideals, in part, because they perceive their partner as more responsive to their sexual needs, but not because of general responsiveness.

\section{Follow-up Analyses}

We also tested whether couples' reports of unmet sexual ideals over the course of the 21day daily experience study predicted changes in their sexual satisfaction and relationship quality three months later, and whether this was moderated by baseline reports of sexual communal strength. These analyses allow us to test if unmet sexual ideals in daily life are associated with declines in sexual and relationship quality over time. Further, this allows us to assess whether having a partner who is more sexually communal can help buffer the negative associations between unmet sexual ideals and sexual and relationship quality over time. To test these longitudinal effects, we created two aggregate variables - one for each partner's unmet sexual 
ideals over the course of the 21-day study - and entered them as simultaneous predictors of participants' sexual satisfaction, relationship satisfaction, and commitment measured three months after they completed the diary study, controlling for these same variables at background. We then included baseline ratings of each partner's sexual communal strength, all interactions between both partner's unmet sexual ideals and sexual communal strength, and entered these as simultaneous predictors of sexual satisfaction, relationship satisfaction, and commitment (assessed separately).

After accounting for the outcome at baseline, people who reported more unmet sexual ideals over the course of the 21-day daily experience study reported declines in sexual satisfaction $(b=-.51, t(191)=-7.40, p<.001,95 \%$ CI $[-.64,-.37])$, relationship satisfaction $(b=$ $-.15, t(195)=-2.92, p=.004,95 \%$ CI $[-.25,-.05])$, and commitment $(b=-.17, t(202)=-4.43, p<$ $.001,95 \%$ CI [-.24, -.09]) three months later (see Table 8). Their partner also reported declines in sexual $(b=-.18, t(187)=-2.73, p=.007,95 \% \mathrm{CI}[-.31,-.05])$ and relationship satisfaction $(b=-$ $.42, t(193)=-8.54, p<.001,95 \%$ CI $[-.51,-.32])$ three months later, but not lower commitment $(p=.214)$

One of the overtime associations was moderated by partner's sexual communal strength (see Table 9; Fig. 5). The association between unmet sexual ideals over the course of the study and a person's own sexual satisfaction three months later was moderated by their partner's sexual communal strength $(b=.22, t(191)=2.34, p=.020,95 \%$ CI $[.03, .40])$. That is, the effect of unmet sexual ideals during the 21-day daily experience study on sexual satisfaction three months later was attenuated, although still significant, among individuals who had partners who were higher in sexual communal strength $(b=-.34, t(183)=-3.62, p<.001,95 \%$ CI $[-.52,-.15])$, compared to people with partners low in sexual communal strength $(b=-.59, t(191)=-7.11, p<$ 
$.001,95 \%$ CI [-.75, -.42]; See Figure 3). However, the association between unmet sexual ideals over the course of the study and a person's own relationship satisfaction $(p=.812)$ and commitment $(p=.401)$ three months later was not moderated by their partner's sexual communal strength. This suggests that unmet sexual ideals were associated with decreased sexual satisfaction, relationship satisfaction, and commitment three months later, but that having a partner higher in sexual communal strength only buffered the effects for sexual satisfaction. Table 9

APIM Models with Daily Unmet Sexual Ideals (USI) and Baseline Sexual Communal Strength (SCS) Predicting Sexual and Relationship Quality Three Months Later Controlling for Outcomes at Baseline-Study 3

$\begin{array}{ccc}\text { Relationship } & \text { Sexual } & \text { Commitment } \\ \text { Satisfaction } & \text { Satisfaction } & \end{array}$

\begin{tabular}{lllllll}
\hline$b$ & $S E$ & $b$ & $S E$ & $b$ & $S E$
\end{tabular}

\begin{tabular}{lcccccc}
\hline Fixed Effects & 5.94 & $.09 * * *$ & 5.85 & $.08 * * *$ & 6.55 & $.07 * * *$ \\
Intercept & -.11 & $.01 *$ & -.46 & $.07 * * *$ & -.21 & $.04 * * *$ \\
Actor (A) USI & -.39 & $.05 * * *$ & -.18 & $.07 * *$ & -.04 & .04 \\
Partner (P) USI & .01 & .05 & .15 & $.06 *$ & .02 & .04 \\
A SCS & .12 & .14 & .25 & .16 & .34 & .11 \\
P SCS & .18 & .14 & .10 & .16 & .004 & .11 \\
A USI x A SCS & .17 & $.08 *$ & .02 & .11 & .07 & .07 \\
A USIs x P SCS & .02 & .07 & .22 & $.10 *$ & -.05 & .06 \\
P USI x A SCS & -.0009 & .07 & .03 & .09 & -.04 & .06 \\
P USI x P SCS & & & & & & \\
\end{tabular}


Note: ${ }^{* * *} p<.001,{ }^{* *} p<.01, * p<.05 .,{ }^{1}$ indicates $\mathrm{p}$-value is marginal at $p=.05-.07$.

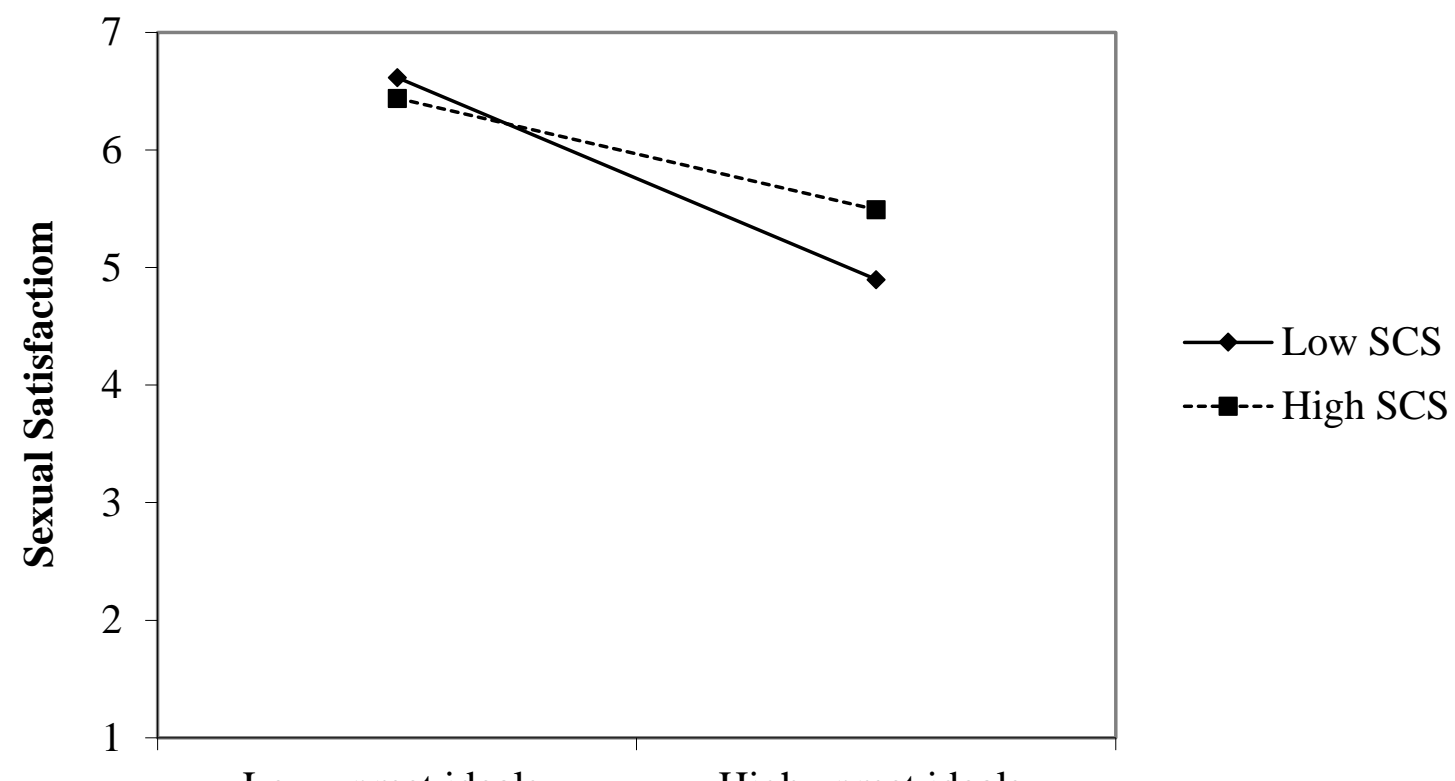

Low unmet ideals High unmet ideals

Fig. 5. The interactive effect of actor's daily unmet sexual ideals and partner's baseline sexual communal strength (SCS) on actor's sexual satisfaction 3-months later controlling for baseline sexual satisfaction (Study 3).

\section{Providing Evidence for Generalizability and Ruling Out Alternative Explanations of the}

\section{Findings}

As in Study 2, we aimed to isolate the direction of the daily diary effects by accounting for the previous day's outcome variable. Accounting for people's sexual and relationship quality on the previous day, on days when people reported greater unmet ideals than they did on average, they reported lower sexual $(b=-.26, t(3560)=-25.42, p<.001,95 \%$ CI [-.28, -.24$])$ and relationship satisfaction $(b=-.20, t(3653)=-21.60, p<.001,95 \%$ CI $[-.22,-.19])$, and poorer commitment $(b=-.09, t(3483)=-11.92, p<.001,95 \% \mathrm{CI}[-.11,-.08])$, and their partner's reported less sexual satisfaction $(b=-.08, t(3560=-8.01, p<.001,95 \%$ CI $[-.10,-.06])$, 
relationship satisfaction $(b=-.06, t(3653)=-6.22, p<.001,95 \%$ CI $[-.08,-.04])$, and commitment $(b=-.02, t(3488)=-2.00, p=.046,95 \%$ CI $[-.03,-.00])$.

Additionally, when controlling for the previous day's outcome, the association between unmet sexual ideals and sexual satisfaction $(b=.09, t(3894)=5.33, p<.001,95 \%$ CI $[.06, .12])$ was buffered by the partner's sexual communal strength. That is, people with partners low in sexual communal strength reported significantly lower sexual satisfaction on days when they had higher unmet sexual ideals $(b=-.30, t(3641)=-20.69, p<.001,95 \%$ CI $[-.33,-.27])$, but the association between daily unmet sexual ideals and lower sexual satisfaction was significantly attenuated among people with partners who were higher in sexual communal strength $(b=-.20$, $t(3610)=-14.22, p<.001,95 \%$ CI $[-.23,-.17])$ when controlling for sexual satisfaction the previous day. However, the association between unmet sexual ideals and a person's own relationship satisfaction $(p=.115)$ and commitment $(p=.131)$ was not moderated by their partner's sexual communal strength when controlling for the previous day's outcome (which is consistent with our findings assessing the moderating role of sexual communal strength without the prior day's outcome). Additionally, the association between unmet sexual ideals and a person's own sexual satisfaction $(p=.559)$, relationship satisfaction $(p=.609)$ and commitment $(p=538)$ was not moderated by their own sexual communal strength when controlling for the previous day's outcome..$^{5}$

Furthermore, as in Study 2, we wanted to rule out the possibility that sexual frequency was driving the effects. To test this, in exploratory analyses we re-ran the daily and follow-up analyses controlling for couples' sexual frequency, and all of the significant effects reported above remained significant. In this study we also wanted to rule out the possibility that the

\footnotetext{
${ }^{5}$ As in Study 2, the effects were constrained to the same day, null effects emerged for next day lagged effects.
} 
buffering effects were driven by a person's level of desire. Given that sexual communal strength has been shown to be associated with higher desire (Muise et al., 2013), we re-ran the analyses controlling for sexual desire, and all significant effects remained significant.

\section{Discussion}

Consistent with our previous findings, the results of Study 3 suggest that on days when individuals experience greater unmet sexual ideals, they report poorer sexual and relationship quality, as do their partners. However, while the association between unmet sexual ideals and sexual satisfaction was weakened when individuals had partners who were high in sexual communal strength, inconsistent with Studies 1 and 2, the buffering effect of having a communal partner did not extend to relationship satisfaction or commitment in this study. Furthermore, results suggest that the effects of unmet sexual ideals endure over time, with greater unmet sexual ideals over the course of the 21-day diary study predicting decreases in sexual satisfaction, relationship satisfaction, and commitment from background to the three-month follow-up. Similar to the daily findings, we found that having a partner who is high in sexual communal strength buffered people against declines in their sexual satisfaction over time due to having unmet sexual ideals but did not buffer against declines in relationship satisfaction or commitment. This provides the first evidence that having a sexually communal partner might help people maintain sexual satisfaction over time, even in the face of unmet ideals. Across the studies thus far we see that the effects are strongest and most consistent for sexual satisfaction; thus, it is possible that the domain-specific effects are the strongest, but there may be more nuance in when and how these effects extend to broader relationship outcomes. Additionally, we assessed whether sexual responsiveness accounted for the moderation between people's unmet sexual ideals and their partner's sexual communal strength in predicting sexual and relationship 
quality, and found that people with partners who are higher in sexual communal strength are buffered against experiencing lower daily sexual and relationship quality in response to having unmet sexual ideals in part because they perceive their partner as more responsive to their sexual needs (though not their general responsiveness).

\section{Study 4}

Studies 1-3 demonstrate that unmet sexual ideals are consistently associated with lower sexual and relationship quality; yet, across these studies having a partner who was highly responsive to one's sexual needs tended to buffer the negative associations between unmet sexual ideals and sexual and relationship quality. However, because the previous studies employed correlational designs, we cannot make causal claims. Although we expect unmet sexual ideals to be driving the effects on sexual and relationship quality, it is also possible that, for example, less satisfied people are more likely to report unmet sexual ideals or that having one's sexual ideals met makes it easier for a partner to be responsive. The goal of Study 4 was to extend these findings by examining unmet sexual ideals and perceived partner sexual communal strength using an experimental design. Experimentally manipulating these constructs, as opposed to examining trait measures, enhances our ability to make causal claims about the role of having a sexually communal partner in buffering against lower relationship and sexual quality among those with unmet ideals, and to rule out the possibility that the associations with relationship quality are driven by other unmeasured variables. We designed an experiment in which people were first assigned to engage in a task that led them to see their partner as either more responsive to their sexual needs (i.e., high in sexual communal strength), or less unresponsive to their sexual needs (i.e., low sexual communal strength), or they were assigned to a control group. Next, they were given false feedback that indicated that they were either compatible (i.e., their partner 
meets their sexual ideals) or incompatible (i.e., their partner does not meet their sexual ideals) with their romantic partner.

Therefore, this study was a 3 (high partner sexual responsiveness, low partner sexual responsiveness, control) by 2 (unmet sexual ideals, met sexual ideals) design. We expected that people who were made to believe their sexual ideals were unmet by their partner would report lower sexual and relationship quality compared to people made to believe their sexual ideals were met. However, our key prediction was that among those manipulated to have unmet sexual ideals, they would report higher sexual and relationship quality when they also believed their partner was highly responsive to their sexual needs, compared to those who perceived their partner to be low in sexual responsiveness or compared to the control condition. In other words, we predicted that individuals who were led to believe that their partner was responsive to their sexual needs would be buffered against the lower sexual and relationship quality that accompanies unmet (compared to met) sexual ideals. All of the predictions were pre-registered on the OSF prior to collecting data.

\section{Method}

\section{Participants and Procedure}

A pre-registered power analysis indicated that 618 participants would be needed to estimate a small to medium effect $(f=.18$ ) with $95 \%$ power (power estimated using G-Power 3.1; Erdfelder, Faul, \& Buchner, 1996; Faul, Erdfelder, Buchner, \& Lang, 2009). To account for incomplete surveys, suspicion, and attrition, we sought to collect at least $25 \%$ more participants than the target sample size, though when we received the data, we were slightly over our quota and resulted in $\sim 29 \%$ over the target sample size. We recruited 799 individuals from the United States, Canada, and Europe through Prolific (https://www.prolific.co/), an online crowdsourcing 
platform that is commonly used for psychological research. Individuals who were at least 18 years old, who had been in a romantic relationship for at least six months, residing in the United States, Canada, or Europe, who are able to read and write in English, and who had access to a computer were eligible to participate. Based on our pre-registered criteria, data from 181 individuals (22.65\%) were excluded because they incorrectly identified the compatibility feedback they were provided or did not remember what it was $(N=99,12.4 \%)$, they indicated that they were suspicious and did not believe the feedback they were provided $(N=42,5.26 \%)$, or they viewed the feedback for less than 10 seconds $(N=40,4.9 \%)$. Additional information about the suspicion and manipulation checks can be found below. The final sample $(N=618)$ was mostly females (66.0\%), who were dating (47.4\%) or married (46.0\%), Caucasian (90.6\%), heterosexual (86.6\%), and monogamous (97.7\%). On average, most participants were in their mid 30s $(M=34.80$ years old, $S D=15.19)$ and were in long-term relationships $(M=5.99$ years, $S D=3.40$ ). See Table 1 for more information about the demographics.

Participants who met the study's criteria and consented to participate were told that the purpose of this study was to learn more about people's romantic and sexual experiences. After providing consent, participants were told that research has shown that people can be grouped into different categories based on their responses to a series of questions that they would answer. We then told participants that we would perform such groupings based on their responses to these questions and would give them feedback later in the study. Participants then answered a series of compatibility questions, which included questions about their sexual ideals, the extent to which they perceived unmet sexual ideals in their current relationship, and their perception of their partner's sexual ideals. After completing the compatibility questions, participants were asked to provide their demographic information. After the demographic questions, the participants were 
then exposed to a perceived partner sexual communal strength manipulation, followed by the sexual compatibility manipulation. That is, this study involved two manipulations:

1. We manipulated perceived partner sexual communal strength (adapted from Lemay, Clark, \& Feeney, 2007; Reis, Lee, O’Keefe, \& Clark, 2018) by asking participants to either list two ways that their partner was attentive to their sexual needs or preferences in the last month (high sexual communal strength), to list 10 ways their partner was attentive to their sexual needs (low sexual communal strength), or to list five things their partner carries around with them (control condition). After participants completed the demographic items, they were randomly assigned to one of these three conditions. The logic of this manipulation-based on a general approach developed by Schwarz and colleagues (1991) — is that it should be easy to recall 2 things that a partner has done, which should activate feelings that a partner is highly responsive to one's sexual needs. However, recalling 10 examples should be more difficult, and this difficulty should lead participants to perceive that their partner is not as responsive to their sexual needs. In fact, in a pilot study of 186 people recruited on Prolific, those in the high sexual communal strength condition did perceive their partner as more sexually responsive compared to those in the low condition $(p=.020)$, but did not significantly differ from those in the control condition ( $p=.337$; See Supplemental Materials).

2. After completing the sexual communal strength task and rating its difficulty, participants were given false feedback about how well their partner meets their sexual ideals in the form of a compatibility score. That is, adapting a previous false feedback manipulation used by Maxwell and colleagues (2017), participant were randomly assigned to one of two conditions wherein they were given feedback based on the sexual ideal compatibility questions they completed, and were told that these results were based on an algorithm that has been well- 
established in the field. In the unmet sexual ideals condition, we told participants that the results show that their partner does not meet their sexual ideals and their sexual compatibility is low (36th percentile), which means that $64 \%$ of individuals who completed similar questions reported that their sexual ideals were met more than theirs (a visual was provided to display this percentile). Participants in this condition were further told that this suggests that they have very different sexual ideals and preferences, and they are likely to face sexual issues and disagreements over time. In the met sexual ideals condition, participants were told that their partner meets their sexual ideals and their sexual compatibility is high (86th percentile) based on an algorithm that has been well-established in the field. Participants in the high condition were told that this means that only $14 \%$ of individuals who completed similar questions reported that their sexual ideals were met more than theirs (a visual was provided to display this percentile). Participants were further told that based on our previous work, the participant and their partner are on the same page sexually and will likely enjoy sexual compatibility over the course of their relationship. Importantly, this feedback was not based on their actual answers, rather participants were randomly assigned to either the high or low compatibility conditions.

Finally, participants were asked to complete several manipulation checks, and a series of questions about their relationship quality (e.g., sexual and relationship satisfaction, commitment), and at the very end of the survey participants were asked suspicion check questions. Upon completion, participants were given a debriefing form that provided more information about the purpose of the study and were asked to provide post-debriefing consent to participation (e.g., to provide final consent to use the data that had already been collected due to the deceit employed in this study). The survey took approximately 10-15 minutes, and as compensation for taking part in this study, individuals were provided \$1.59 USD (e.g., £1.25 
GBP; $€ 1.40$ EUR; \$2.09 CAD). For more information about the study’s measures, please view the measures document on the $\underline{\mathrm{OSF}}$.

\section{Manipulation and Suspicion Checks}

\section{Perceived Partner Sexual Communal Strength Manipulation Check. Two}

manipulation checks were included for the perceived partner sexual communal strength manipulation. First, immediately after completing the sexual communal strength manipulation (described above) participants were asked to indicate how difficult the task was on a 7-point scale $(1=$ not at all, 7 = very difficult $)$. The idea is that it should be relatively difficult to recall 10 things a partner has done to be sexually responsive, whereas it should be relatively easy to recall two things a partner has done to be sexually responsive, or five things a partner carries around with them. To assess the effectiveness of the perceived partner sexual communal strength manipulation, reports of difficulty of the task (assessed immediately after the sexual communal strength manipulation) and reports of perceived partner sexual communal strength at the conclusion of the study were assessed using a between subjects ANOVA in which the perceived partner sexual communal strength experimental condition (high perceived partner sexual communal strength versus low perceived partner sexual communal strength versus control) was the predictor, and the outcome measures included the difficulty rating and the perceived partner sexual responsiveness measure at the conclusion of the study. As intended, a main effect for the difficulty of the task condition emerged, $F(616)=51.32, p<.001, \eta_{p}{ }^{2}=.14$. Those in the low perceived partner sexual communal strength condition $(M=4.33, S E=0.14)$ perceived the recall task as more difficult than those in the high partner perceived sexual communal strength condition $(M=2.90, S E=0.14 ; t(616)=7.26, p<.001,95 \%$ CI $[0.96,1.90])$ or the control condition $(M=2.46, S E=0.13 ; t(616)=9.73, p<.001,95 \%$ CI $[1.41,2.33])$, while those in 
high perceived partner sexual communal strength condition did not differ from the control $(p=$ .073). Importantly, the mean score in the low sexual communal strength condition was at the midpoint $(M=4.33$, on the 1-7 scale used; which is consistent with past research that used a similar manipulation, see Reis et al., 2018), which suggests that participants did not have or did not acknowledge having difficulty generating 10 things a partner had done to be responsive to their sexual needs, or that partners were relatively high in sexual communal strength rendering this task easier than expected — though it is worth noting this task was still more difficult than listing two things a partner has done to be responsive, or to listing five things a partner carries around with them.

Second, participants were asked to rate two items that assessed their perception of their partner's sexual communal strength post-manipulation (e.g., "My partner is motivated to meet my sexual needs" and "My partner understands my sexual needs") on a 7-point scale (1 = do not agree at all, $7=$ agree completely). These two items were mean aggregated with higher scores indicating higher perceived partner sexual communal strength $(\alpha=.87, M=5.41, S D=1.60)$. To further assess the effectiveness of the perceived partner sexual communal strength manipulation, reports of perceived partner sexual communal strength (assessed after both manipulations were complete) were assessed using a between subjects ANOVA, as was done above, though the outcome measure was now perceived partner sexual communal strength measure at the conclusion of the study (assessed separately). Across the perceived partner sexual communal strength conditions, people differed in their perceptions of their partner's sexual communal strength, $F(617)=3.26, p=.039, \eta_{p}{ }^{2}=.01$. That is, people in the high perceived partner sexual communal strength condition $(M=5.64, S E=0.11)$ reported that their partner was more sexually communal post-manipulation compared to those in the low partner sexual communal strength 
condition $(M=5.25, S E=0.11, t(617)=2.47, p=.043,95 \%$ CI $[.01, .77])$, but did not significantly differ from those in the control condition $(M=5.35, S E=11 ; p=.188)$, while those in the low perceived sexual communal strength condition also did not differ from those in the control $(p=1.00)$. That is, the results suggest that those in the high sexual communal strength condition found the task to be easier and rated their partner as higher in sexual communal strength post-manipulation compared to those who were in the low sexual communal strength condition, but not compared to the control condition.

Unmet Sexual Ideal Manipulation Check. For the unmet sexual ideal manipulation, a suspicion check, attention check, and manipulation check (one item for each) were included. First, immediately after being provided with the sexual compatibility feedback (described above) participants were told that we wanted to make sure the feedback they received was clear, and they were asked via an open response question to tell us what the results said about the extent to which their partner is compatible with their sexual ideals and if they were not sure they could write "I don't know." When participants indicated that they were not sure, misidentified the feedback provided, or indicated that the feedback was false, their data was excluded from the analyses. To decipher this, all responses provided by participants were independently rated by two of the investigators. Any disagreements were resolved through discussion, and when necessary, a collaborator on the project was asked to weigh in. Kappa's (Cohen, 1960) agreement between raters was .84 , indicating high agreement between coders (e.g., McHugh, 2012). ${ }^{6}$ Second, when participants were provided the compatibility feedback, the amount of time they viewed the information was timed. Those who had a time of 10 seconds or less would not

\footnotetext{
${ }^{6}$ Cohen (1960) suggested the Kappa result be interpreted as follows: values $<0$ as indicating no agreement and 0.01-0.20 as none to slight, 0.21-0.40 as fair, 0.41- 0.60 as moderate, 0.61-0.80 as substantial, and 0.81- 1.00 as almost perfect agreement.
} 
have had enough time to read the feedback and were excluded from the study. ${ }^{7}$ Third, participants were asked to rate their sexual compatibility with their partner post-manipulation (e.g., "My partner and I are sexually compatible") on a 7 -point scale $(1=$ do not agree at all, $7=$ agree completely; $M=5.40, S D=1.65)$. This allowed us to assess whether the manipulation was effective, but it was not used to exclude participants. To assess the effectiveness of the sexual ideal compatibility manipulation, reports of perceived unmet sexual ideals at the conclusion of the study were assessed using a between subject ANOVA wherein the sexual compatibility experimental condition (met versus unmet sexual ideals) was the predictor, and the outcome measure consisted of participants self-reported compatibility at the conclusion of the study. Results suggest that across the unmet sexual ideals conditions, people differed in their perceptions of their sexual compatibility post-manipulation, $F(617)=77.96, p<.001, \eta_{p}^{2}=.11$. Those who were told that their sexual ideals were unmet by their partner $(M=4.82, S E=0.09)$ reported lower sexual compatibility with their partner (post-manipulation) than those who were told that their sexual ideals were met by their partner $(M=5.93, S E=0.09 ; t(617)=-8.83, p<$ $.001,95 \%$ CI $[-1.35,-0.86])$.

Suspicion Checks. After completing all other measures, participants were asked two open ended questions to gauge their suspicion about the study overall (e.g., "Do you have any thoughts about what we might be looking for in this study?"; "Do you have any thoughts you'd like to share about the study?"). Participants who indicated that the compatibility feedback provided was false, or similar responses indicating suspicion, were excluded from analyses. To decide this, all responses provided by participants regarding their suspicion were independently

\footnotetext{
7 The exclusion criteria were pre-registered prior to collecting data and were established based on the pilot study (see the OSF for more information).
} 
rated by two of the investigators, as was done with the sexual ideal manipulation, with Kappa's (Cohen, 1960) agreement between raters (.63) indicating substantial agreement between coders (e.g., McHugh, 2012).

\section{Outcome Measures}

Sexual Satisfaction. A single item (e.g., "I feel satisfied with our sexual relationship") was used to assess sexual satisfaction. Possible responses were on a 7-point scale $(1=$ do not agree at all, $7=$ agree completely; $M=5.53, S D=1.39)$.

Relationship Satisfaction. A single item (e.g., "I feel satisfied with our romantic relationship") was used to assess relationship satisfaction. Possible responses were on a 7-point scale $(1=$ do not agree at all, $7=$ agree completely; $M=6.06, S D=1.18)$.

Commitment. A single item (e.g., "I am committed to maintaining my relationship with my partner") was used to assess commitment among couples. Possible responses were on a 7point scale $(1=$ do not agree at all, $7=$ agree completely; $M=6.57, S D=0.90)$.

\section{Results}

Effects of Unmet Ideals and Perceived Partner Sexual Communal Strength on Sexual and

\section{Relationship Quality.}

To assess our primary hypothesis, we examined whether reports of sexual satisfaction and relationship quality significantly differed by the experimental conditions. More specifically, we conducted 3 (perceptions of a partner as high in sexual responsiveness versus low versus control) by 2 (met versus unmet sexual ideals) between-subjects ANOVAs wherein experimental conditions and the interaction between the experimental conditions were the independent variables, and the outcome measures consisted of sexual satisfaction, relationship satisfaction, and commitment, tested in separate models. As with the previous studies, in exploratory analyses 
we further assessed whether gender affected the manipulations' impact on sexual and relationship quality. The analytic plan and the data and syntax for these analyses can be found on the OSF. Correlations between all variables are in Table 10 and descriptive statistics for all comparisons reported below can be found in Table 11.

Table 10

Correlations Among Focal Variables (Post-Manipulation) for Study 4

\begin{tabular}{|c|c|c|c|c|c|}
\hline & 1 & 2 & 3 & 4 & 5 \\
\hline 1. Relationship Satisfaction & - & & & & \\
\hline 2. Sexual Satisfaction & $.62 * * *$ & - & & & \\
\hline 3. Commitment & $.48 * * *$ & $.34 * * *$ & - & & \\
\hline 4. Sexual Communal Strength & $.49 * * *$ & $.70 * * *$ & $.34 * * *$ & - & \\
\hline 5. Perceived Sexual Ideal & $.52 * * *$ & $.68 * * *$ & $.42 * * *$ & $.72 * * *$ & - \\
\hline Compatibility & & & & & \\
\hline
\end{tabular}

Note: $* * * p<.001, * * p<.01, * p<.05$. Post-manipulation reports of specific variables were included in correlations.

Results of the ANOVAs showed that across the unmet sexual ideals conditions people differed in their reports of sexual satisfaction $\left(F(617)=12.49, p<.001, \eta_{p}{ }^{2}=.02\right)$, relationship satisfaction $\left(F(617)=11.80, p=.001, \eta_{p}^{2}=.02\right)$, and commitment $\left(F(617)=7.34, p=.007, \eta_{p}{ }^{2}\right.$ $=.01)$ post-manipulation. Those who were told that their sexual ideals were unmet by their partner reported lower sexual and relationship quality (post-manipulation) than those who were told that their sexual ideals were met by their partner (sexual satisfaction, $t(617)=-3.52, p<$ $.001,95 \%$ CI [-.61, -.17]; relationship satisfaction, $t(617)=-3.45, p=.001,95 \%$ CI [-.50, -.14]; and commitment, $t(617)=-2.72, p=.007,95 \%$ CI [-.34, -.54]). 
Additionally, across the perceived partner sexual communal strength conditions reports differed for sexual satisfaction $\left(F(617)=5.01, p=.007, \eta_{p}{ }^{2}=.02\right)$ and relationship satisfaction $\left(F(617)=7.98, p<.001, \eta_{p}{ }^{2}=.03\right)$ post-manipulation. That is, people in the high perceived partner sexual communal strength condition reported higher sexual and relationship satisfaction post-manipulation, compared to those in the low partner sexual communal strength condition (sexual satisfaction, $t(617)=2.99, p=.009,95 \%$ CI $[.08, .74]$; relationship satisfaction, $t(617)=$ $3.86, p<.001,95 \% \mathrm{CI}[.17, .73]$ ), and compared to the control condition (sexual satisfaction, $t(617)=2.47, p=.042,95 \%$ CI $[.01, .66]$; relationship satisfaction, $t(617)=2.83, p=.014,95 \%$ CI $[.05, .60])$. However, those in the low partner sexual communal strength condition did not differ from those in the control condition for sexual $(p=1.00)$ or relationship $(p=.675)$ satisfaction. Furthermore, there was no main effect of perceived partner sexual communal strength for commitment (all $p s>.05$ ).

Table 11

Comparing Sexual and Relationship Quality across Sexual Communal Strength and Sexual Ideal Conditions in Study 4
High SCS
Low SCS
Control

\section{Variables}

$M \quad S E \quad M$

$S E$

$\boldsymbol{t}$

M

SE

$t$

\section{Unmet Sexual Ideals}

\section{Condition}

1. Sexual Satisfaction

5.78

0.14

5.12

0.14

$3.35^{* *}$

5.10

0.14

$3.47 * *$

2. Relationship Satisfaction

6.33

0.12

5.59

0.12

$4.44 * * *$

5.79

0.12

$3.27 * *$

3. Commitment

$6.51 \quad 0.09$

6.40

0.09

0.85

6.51

0.09

0.00 
1. Sexual Satisfaction 5.77

0.14

5.61

0.13

0.83

5.78

0.13

0.05

2. Relationship Satisfaction

6.31

0.12

6.15

0.11

0.99

6.20

0.11

0.69

3. Commitment

$6.74 \quad 0.09$

6.69

0.09

0.44

6.58

0.08

1.26

Note: $* * * p<.001, * * p<.01, * p<.05$.

\section{The Buffering Effect of Perceived Partner Sexual Communal Strength}

Consistent with our pre-registered predictions, the main effects were qualified by a sexual ideals (met versus unmet) by perceived partner sexual communal strength (low perceived sexual communal strength versus high perceived partner sexual communal strength versus control) interaction for sexual $\left(F(617)=3.38, p=.035, \eta_{p}^{2}=.01\right.$, see Figure 6A) and relationship satisfaction $\left(F(617)=3.35, p=.036, \eta_{p}^{2}=.01\right.$, see Figure $\left.6 \mathrm{~B}\right)$. However, there was no significant interaction for commitment $(p=.461)$. For participants who were told that their partner meets their sexual ideals, sexual and relationship satisfaction did not differ based on perceived partner sexual communal strength (high, low, control; all $p>.05$ ). However, among participants who were told they have unmet sexual ideals, sexual and relationship satisfaction was significantly higher in the high perceived partner sexual communal strength condition compared to the control condition (sexual satisfaction: $t(617)=3.47, p=.002,95 \% \mathrm{CI}[.21$, 1.14]; relationship satisfaction: $t(617)=3.27, p=.003,95 \% \mathrm{CI}[.14, .93])$ and the low perceived partner sexual communal strength condition (sexual satisfaction: $t(617)=3.28, p=.003,95 \% \mathrm{CI}$ $[.19,1.13]$; relationship satisfaction: $t(617)=4.44, p<.001,95 \%$ CI $[.34,1.14])$. However, no differences were found among those in the low and control conditions (all $p>.05$ ). Put another way, people who perceived their partner as high in sexual communal strength reported the same levels of sexual and relationship satisfaction when they were told they had unmet sexual ideals as 
when their sexual ideals were met (sexual satisfaction, $p=.981$; relationship satisfaction, $p=$ .932).

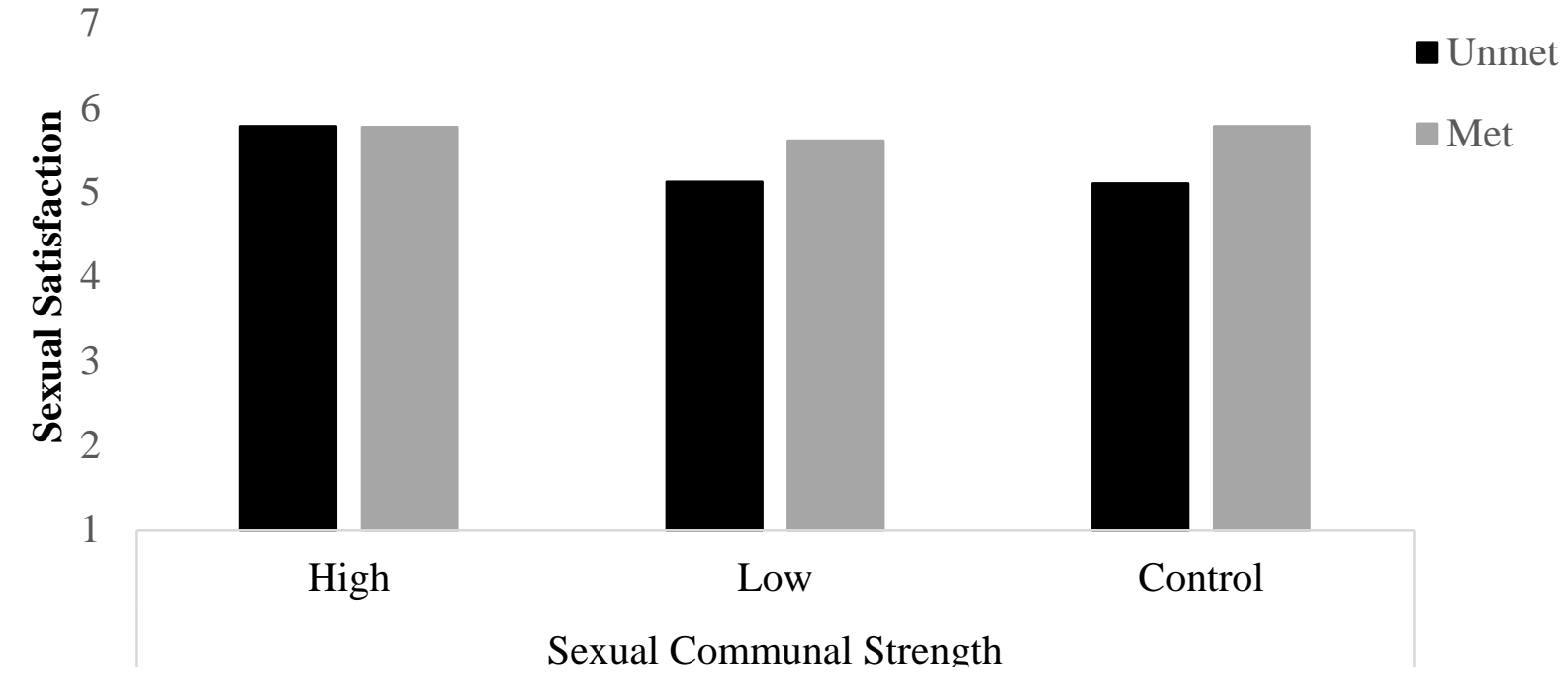

Fig. 6A. Mean Ratings Across Perceived Partner Sexual Communal Strength and Sexual Ideal Conditions for Sexual Satisfaction (Study 4).

7

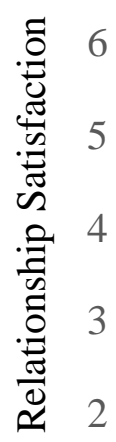

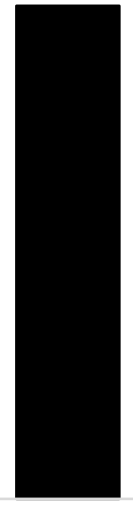

High

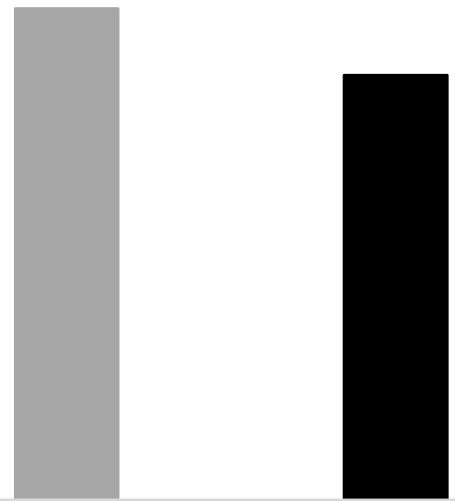

Low

-Unmet

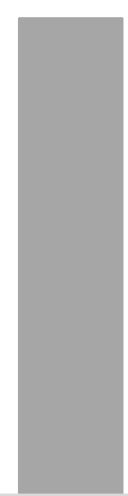

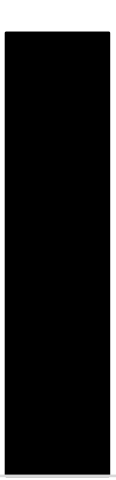

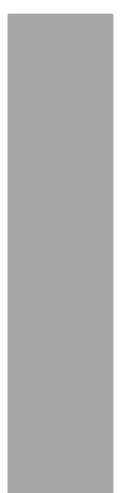

Control

Sexual Communal Strength

Fig. 6B. Mean Ratings Across Perceived Partner Sexual Communal Strength and Sexual Ideal Conditions for Relationship Satisfaction (Study 4). 


\section{Discussion}

In Study 4, we found that those who were told that their sexual ideals were unmet by their partner reported lower sexual and relationship quality (post-manipulation) than those who were told that their sexual ideals were met by their partner. We also found that those who were primed to perceive their partner as more responsive to their sexual needs reported greater sexual and relationship quality (post-manipulation) than those who were led to believe their partner was low in sexual communal strength and to a control condition. This study provides experimental support for the buffering effect of a partner's sexual communal strength. That is, people who perceived their partner as high in sexual communal strength reported the same levels of sexual and relationship satisfaction when they were told they had unmet sexual ideals as when their sexual ideals were met. This pattern of results provided evidence for the buffering effect of having a sexually communal partner on the lower sexual and relationship satisfaction associated with unmet sexual ideals. However, in contrast to our predictions, we did not find support for commitment.

\section{General Discussion}

Across four studies - using cross-sectional, daily diary, dyadic, and experimental methods - we demonstrated that unmet sexual ideals are associated with both partners' lower sexual and relationship quality. However, across studies, having a partner who is high in sexual communal strength — motivated to be responsive to their partner's sexual needs — buffered against the negative associations between unmet sexual ideals and sexual and relationship quality. Across studies, the results were largely consistent across gender (examined in Studies 14) and could not be accounted for by sexual frequency (examined in Studies 2-3) or sexual desire (examined in Study 3). 


\section{Applying the Ideal Standards Model to Sexuality}

Extending previous research on ideal standards in relationships, across the current set of studies, when people reported that their partner did not meet their sexual ideals or were made to believe they had unmet sexual ideals, they reported poorer sexual and relationship quality. In Studies 2 and 3, on days when people reported having more unmet sexual ideals than typical, both they and their partner reported lower sexual and relationship satisfaction and poorer commitment, and, in Study 3, people who reported more unmet sexual ideals over the course of the 21-day daily experience study reported declines in sexual and relationship satisfaction, and were less committed to their partner three months later. Their partner also reported declines in sexual and relationship satisfaction three months later. In Study 4, we provided experimental evidence that people who were given (false) feedback that their sexual ideals were unmet by their partner reported lower sexual and relationship satisfaction (but not commitment) postmanipulation than those who were told that their sexual ideals were met by their partner. These results provide consistent evidence for the detriments of unmet sexual ideals on both individuals and their partner's sexual and relationship quality and are in line with the Ideal Standards Model (Campbell et al., 2013; Campbell et al., 2001; Campbell et al., 2016; Csajbók \& Berkics, 2017; Fletcher et al., 2000; Overall et al., 2006), as well as Interdependence Theory (Kelley \& Thibault, 1978; Rusbult \& Buunk, 1993) and Self-Discrepancy Theory (Higgins, 1987; Higgins et al., 1985), which would posit that relationships should be more satisfying and likely to endure when a romantic partner meets or exceeds one's ideals.

The current findings also corroborate past work showing that discrepancies between a person's perceptions of themselves, their life, their housing conditions, as well as their relationships (more generally), compared to their ideal for that domain is associated with 
domain-specific dissatisfaction or unhappiness (e.g., Baucom et al., 1996; Campbell et al., 2001; Fletcher et al., 1999; Higgins et al., 1985; Kelley \& Burgoon, 1991; Meltzer et al., 2014; Michalos, 1986; Ruvolo \& Veroff, 1997). While prior research has examined discrepancies between a person's ideal standards and their actual experience in various domains, past research had not assessed unmet sexual ideals. Thus, the current study extends previous research by demonstrating that individuals hold ideal preferences for their sexual relationship specifically, and their perceptions of having their sexual ideals met versus unmet, are associated with both sexual and relationship quality in general, in the moment, and over time.

There are several theoretical and methodological advantages to extending the ideal standards model to the domain of sexuality. The current research provides novel insight into what encompasses people's sexual ideals and indicates that sexual ideals can include a variety of preferences and partner attributes. In fact, the array of ideals held for a sexual partner contrasts and extends previous research on sexual discrepancies that has largely focused on differences in specific domains of sexual experiences (e.g., desired frequency of sex, levels of sexual desire, and specific sexual acts that are commonly engaged in; Davies et al., 1999; Dworkin \& O’Sullivan, 2005; Mark, 2012; Santilla et al., 2007). As such, examining individuals' sexual ideals more broadly, rather than limiting the focus on sexual desire or aspects of sexual experiences specifically, may provide a more comprehensive understanding of sexual need fulfillment in relationships.

Beyond extending our understanding of sexual ideals, this current research provides insights into who is more likely to successfully navigate unmet sexual ideals. Indeed, a growing body of research has examined the cognitive tactics that individuals use to sustain their relationships when aspects of their partner are less than ideal, but this past work has focused on 
individual rather than dyadic or relational strategies. For example, individuals frequently reframe their expectations to more closely fit with the reality of their partner (e.g., Fletcher et al., 2000), perceive their partner to more closely resemble their ideal than they actually do (e.g., Murray et al., 1996), mitigate negative partner qualities by associating unfavorable attributes with more virtuous traits (e.g., free-spirited as opposed to unreliable; Murray \& Holmes, 1999), or try to change their partner to meet their ideals (Overall et al., 2006). However, attempts to change one's partner to match their ideals are often unsuccessful (see Hira \& Overall, 2011) and are only able to produce small perceived partner improvements, with greater attempts to change one's partner actually reducing how closely the partner was perceived to match ideal standards, which, in turn, was associated with negative relationship evaluations (Overall et al., 2006). As such, this is one of the first studies to show that the negative consequences of unmet (sexual) ideals can be attenuated (i.e., reduced in significance), and in some cases buffered entirely (i.e., reduced to non-significance) through a partner's motivation to be attuned to and motivated to meet their needs. Our work suggests that aiming to have all ideals met through regulation of one's own ideals, or attempts to change their partners to meet their ideals, which has been the focus of past work, may not be the only option for mitigating the consequences of unmet ideals - rather, a partner's sexual communal strength (Studies 1-3) and one's perception of their partner's sexual communal strength (Study 4) can help people maintain sexual and relationship quality in the face of unmet sexual ideals, and this occurs, in part, because people perceive their partner to be more responsive to their needs (Studies 2-3). It is possible that the findings from the current research could be extended to test whether having a responsive partner more generally might buffer against unmet ideals in other relationship domains.

\section{Buffering Effect of Sexual Communal Strength}


Across the studies we found that having a partner who was attuned to and motivated to meet one's sexual needs (i.e., high in sexual communal strength) can help individuals maintain sexual satisfaction and, in some instances, relationship satisfaction and commitment, even in the face of unmet sexual ideals. Although people with sexually communal partners still experience some degree of unmet sexual ideals, they may be better able to navigate these situations in ways that maintain their satisfaction with their sex life and relationship, compared to people who have partners who are less sexually communal. Additionally, in the current study we explored whether one reason why people with partners higher in sexual communal strength are buffered against feeling lower levels of sexual and relationship quality when the have unmet sexual ideals is because they perceive their partner to be highly responsive generally (Studies 2-3) and in response to their sexual needs specifically (Study 3). We found support for a mediated moderation, such that the moderation between unmet sexual ideals and a partner's sexual communal strength on the daily outcomes (sexual satisfaction, relationship satisfaction, commitment) was mediated by daily perceived partner responsiveness (Study 2) and daily perceived partner sexual responsiveness (Study 3). This corroborates past research that has shown that partners higher in sexual communal strength are perceived as more responsive in general (Muise et al., 2013) and to their partner's needs during sex specifically, and this, in turn, is associated with higher sexual relationship quality (Muise \& Impett, 2015).

When coping with unmet sexual ideals, couples may need to negotiate and at times sacrifice their sexual preferences to satisfy both partners. This process may be facilitated by having a partner who is high in sexual communal strength and by recognizing the enacted care provided by their partner (e.g., perceiving one's partner to be responsive), which could create an environment in which sexual experiences are able to be enjoyed despite unmet sexual ideals. We 
see these results as initial evidence that perceptions of a partner being responsive to one's needs are partially responsible for the buffering effect of a partner's sexual communal strength, however, in future work it will be important to investigate these associations in longitudinal data to understand the time-course of sexual ideals and partner's sexual communal strength in relationships.

There are additional reasons why having a partner high sexual communal strength might buffer the negative consequences of unmet sexual ideals. It is possible that partners who are higher in sexual communal strength are more supportive when declining a partner's sexual advances (see Muise et al., 2017), make more efforts to understand and prioritize a partner's sexual interests (Day et al., 2015), and are more willing to compromise or offer other forms of affection when disinterested in sex (e.g.,. being responsive to partners in non-sexual ways; Kim, Muise, Sakaluk, Rosen, \& Impett, 2020). People higher in sexual communal strength may also be more likely to foster open sexual communication in a relationship. Although this has not been tested empirically, in a qualitative study people reported that one way they meet their partner's sexual needs is by communicating with their partner about their sexual likes and dislikes, and ensuring mutuality, such that both partners' needs are acknowledged and met in the relationship (Muise \& Impett, 2015). Additionally, people who communicate more with their partner about their sexual ideals and who are responsive to their partner's sexual ideals in return, may be more inclined to make changes in their sexual behavior to better meet their sexual ideals (i.e., sexual transformations; Burke \& Young, 2012) or for their partner to favor their preferences (i.e., sexual compliance; Impett \& Peplau, 2003; Katz \& Tirone, 2009).

Interestingly, across all studies, a partner's sexual communal strength was a more consistent buffer of the link between unmet sexual ideals and sexual and relationship quality than 
was a person's own sexual communal strength. In contrast to our predictions, results for the buffering effect of a person's own sexual communal strength were mixed and inconsistent across the studies. In most instances, a person's own sexual communal strength did not buffer the association between unmet sexual ideals on their sexual and relationship quality, and when effects did emerge, some were in the predicted direction (e.g., Study 1's results for commitment and Study 2's results for relationship satisfaction), while others were in the opposite direction (Study 2's results for sexual satisfaction). This is unexpected and intriguing given that previous research has documented consistent benefits of being sexually communal. For example, in crosssectional, daily diary, and longitudinal studies, people who are higher in sexual communal strength report higher sexual satisfaction, sexual desire, relationship satisfaction, and commitment, even at times when couples report different sexual interests or are coping with clinical sexual issues (for a review, see Muise \& Impett, 2015; also see Day et al., 2015; Kogan et al., 2010; Muise et al, 2017).

One explanation for the unexpected finding is that communal people might be more focused on being responsive to their partner's sexual preferences, and this may not always buffer their own unmet ideals or it may even be at the cost of meeting their own ideals. In fact, based on past work, communal people tend to be more focused on the maintenance of the relationship as opposed to meeting their own needs (Impett, Peplau, \& Gable, 2005; Kogan et al., 2010), and may approach their sexual experiences with their partner differently than people who are less communal. For example, people high in sexual communal strength tend to focus on the positive aspects of their sexual experiences (Impett, Muise, \& Harasymchuk, 2018), provide care to enhance their partner's welfare and not for their own benefit (Canevello \& Crocker, 2010), believe that sexual relationships take work and effort to thrive (Muise \& Impett, 2016), and are 
understanding and caring when their partner is not in the mood for sex-even if their own desire is high (Kim et al., 2018). Although being motivated to be meet to a partner's sexual needs can be associated with greater sexual and relational quality, if the motivation to meet a partner's sexual needs involves self-neglect — high unmitigated sexual communion - this can instead be associated with poorer sexual and relationship quality (Fritz \& Helgeson, 1998; Impett et al., 2018; Muise et at., 2017; Muise et al., 2018)). We did not assess unmitigated sexual communion in the current set of studies, but it tends to be correlated with sexual communal strength (Muise et al., 2017, 2018), and could be responsible for these unexpected findings.

As such, the role of one's own sexual communal strength in buffering effects could be contingent on other factors. For example, it may depend on the degree to which a partner is sexually communal in response. In the current study, couples showed low concordance in sexual communal strength, such that correlations between partners' levels of sexual communal strength ranged from small to moderate (Study 1, $r=.34, p<.001$ ) to not significant (Study 2, $r=.01$; Study $3, r=-.02$ ). In a recent meta-analysis, partners levels of general communal strength were correlated, but the correlational was small ( $r=.26$; see Le et al., 2018). It is possible that individuals who are motivated to meet their partner's sexual needs but do not receive the same responsiveness in return may perceive their efforts to be exploitive (e.g., see Mills \& Clark, 1986) and may benefit less from their communal efforts. An important avenue for future research is to further explore the importance of concordance in partners' sexual communal strength, additional mechanisms that account for the buffering effect of a partner's sexual communal strength on unmet sexual ideals, and the reverse effects found for an individual's own sexual communal strength on the association between unmet sexual ideals and lower sexual and relationship quality. 


\section{Implications, Limitations, and Future Directions}

The current research suggests that unmet sexual ideals are common and can be detrimental to relationships. Despite the prevalence and negative associations between unmet sexual ideals and sexual and relationship quality, interventions aimed at managing sexual incompatibilities, or incompatibilities in general, are scarce (see Walton, 2014 for a review). The current research provides initial evidence that having a sexually communal partner might help protect against the detriments of unmet sexual ideals. This work also provides initial evidence that perceptions of a partner's sexual communal strength can be manipulated — and even enhanced - through asking people to reflect on recent instances in which their partner was attuned to and motivated to meet their sexual needs. In doing so, people were able to maintain sexual and relationship quality despite perceptions of unmet sexual ideals. Because sexual differences between partners are common (Miller et al., 2003) and among the most difficult types of conflicts to successfully resolve (Sanford, 2003), the findings could have implications for couples who are coping with differing sexual interests, and for clinicians working with these couples. Indeed, based on this research, it seems possible to enhance people's perceptions of their partner's sexual communal strength by having them focus on the ways in which their partner is attentive and responsive to their sexual needs. Given the current and past research findings suggesting that a communal approach to a sexual relationship is associated with greater sexual and relationship quality, even for those with unmet sexual ideals, future work could consider whether it is possible to enhance people's endorsement of sexual communal norms, and if doing so would influence the degree to which a partner meets one's sexual ideals, and could promote sexual and relationship quality. Future work can also consider the extent to which people detect changes in their partner's sexual communal strength and whether a partner's 
motivation to be responsive to their sexual needs has to be accurately detected by a person to have benefits for sexual and relationship quality. Although it is an important caveat that future work should involve both partners to acknowledge that communal strength might be most beneficial when both partners provide communal care to each other and when communal giving does not involve self-neglect.

In this work we focused on perceptions of unmet sexual ideals, but it remains unknown whether correspondence between a person's sexual ideals and their partner's actual traits and preferences - that is, between a person's ratings of their sexual ideals and their partner's ratings of themselves on those same traits and preferences - influences sexual and relationship quality. Past approaches to comparing partner ideal-actual match has been limited by statistical biases, such as correlating difference scores with the outcome variable-which is not the ideal approach for testing matching effects (Cronbach \& Furby, 1970; Edwards, 1994; Edwards, 2001; Edwards \& Parry, 1993). The limitations of the ways in which ideal-actual matching has been tested in previous research have contributed to inconsistent findings in the effects of ideal-actual match in relationship ideals (for a review, see Eastwick et al., 2019). However, advances in statistical analyses for testing matching effects (e.g., response surface analysis; Humberg, Nestler, \& Back, 2018; Nestler, Grimm, \& Schönbrodt, 2015; Schönbrodt, 2016) can provide a powerful alternative approach that overcomes the limitations of past work and can enable researchers to test how and under what conditions ideal-actual match in sexual ideals are predictive of sexual and relationship quality among couples. Similarly, as the results suggest that correspondence in partners' sexual communal strength is low, and we believe this could be one of the contributing factors to the null and at times reverse effects for the moderating effect of an individual's own sexual communal strength, an important future direction is to examine the matching effects of 
sexual communal strength to assess whether mismatched levels of sexual communal strength in a relationship, especially for individuals with unmet sexual ideals, is associated with lower sexual and relationship quality in response to unmet sexual ideals.

The current studies focused on the associations of unmet sexual ideals and sexual and relationship quality among couples in long-term relationships, but it remains unknown whether ideal sexual preferences guide relationship formation processes, and whether ideal-actual match in the early stages of a relationship influences sexual and relationship quality over time. Future research could ask individuals about their ideal sexual preferences when they are single (i.e., not currently romantically attached) or upon entering a relationship, and could assess self-evaluations of a new romantic partner as well the partner's actual rating of themselves across the same traits and attributes over time (similar designs used by Campbell et al., 2016; Gerlach et al., 2019). This would allow researchers to explore whether individuals enter new relationships with others who match their ideal preferences, and if the degree of match between one's ideal preferences and the qualities of their partner predicts relationship formation and maintenance over time. For example, research has shown that relationships develop and can be maintained more smoothly and successfully when individuals enter relationships with a partner who more closely matches their ideal preferences (Campbell et al., 2016; Gerlach et al., 2019), individuals are more satisfied in relationships when they perceive smaller discrepancies between their ideal standards and partner perceptions (Buyukcan-Tetik et al., 2017; Campbell, et al., 2001; Fletcher et al., 1999; Fletcher et al., 2000), and peoples' ideal standards for a romantic relationship can change to become more in line with their perception of their partner's over the first year together (Fletcher et al., 2000)—making these worthwhile avenues to extend to sexual ideals in future work. 
Finally, this research examines unmet sexual ideals and the role of sexual communal strength and perceived partner responsiveness among a relatively homogenous sample of mostly mixed-sex couples, and thus these findings should only be generalized to a population similar to the sample itself: a primarily white, heterosexual, long-term, monogamous sample of couples. Despite our efforts to address some relevant demographic factors in the scale development, we did not have enough diversity in race and other important demographic factors (e.g., age, relationship orientation) to test whether the association of unmet sexual ideals with sexual and relationship quality were widely generalizable. Additionally, while most of the results were consistent for men and women across the four studies, there may be interesting nuances that go undetected among such a homogenous sample. For example, one of the reasons sexual ideals might be so impactful for couples' sexual and relationship quality is because romantic relationships are often sexually exclusive (Blanchflower \& Oswald, 2004). If a partner does not meet a person's sexual ideals, they may have fewer options for meeting their sexual needs than they would for other types of needs. Looking at the association between unmet sexual ideals and sexual and relationship quality in a sample of individuals in consensually non-monogamous relationships - relationships in which partners explicitly agree that extradyadic romantic or sexual relationships are permitted (Conley, Ziegler, Moors, Matsick, \& Valentine, 2013—would afford the opportunity to examine the relative importance of having ideals met by one partner, as opposed to distributing the fulfillment of ideals across multiple concurrent partners. As such, a worthwhile direction for future research would be to examine whether people in consensually non-monogamous relationships seek out and maintain other concurrent sexual relationships to help meet their sexual ideals, and whether outsourcing the fulfillment of ideals across partners helps buffer against the detriments of unmet sexual ideals in relationships. 


\section{Concluding Remarks}

Managing unmet sexual ideals in a relationship can be a difficult task. In the current studies, we demonstrate that unmet sexual ideals are common among couples in long-term relationships and are negatively associated with evaluations of sexual and relationship quality for the individual and their partner. Unmet sexual ideals were associated with lower sexual and relationship quality when assessed in general, in daily life and over time, and in an experimental design when people were made to believe they had unmet versus met ideals. However, across studies we demonstrate that individuals who had partners who were responsive to their sexual needs (e.g., high in sexual communal strength) were able to maintain sexual and relationship quality in the face of unmet sexual ideals, whereas individuals with a partner low in sexual communal strength reported lower sexual and relationship quality when their sexual ideals were unmet by their partner. The findings have implications for theories of ideal standards in relationships and communal motivation, and for couples navigating unmet sexual ideals. 


\section{Supplemental Materials}

The supplemental materials provide additional information about the Sexual Ideals Scale and about exploratory analyses conducted across the studies. The items for the scale were developed and examined by the current researchers prior to conducting the primary analyses included in this manuscript, and all exploratory questions were tested after the primary analyses were examined. The hypotheses, methods, analytic approach, and results that pertain to the Sexual Ideals Scale and the exploratory analyses not included in the manuscript can be found in the Supplemental Materials.

Supplement 1. Sexual Ideals Measure for Studies 1A-1C: Item Generation, Development and Validation of the Sexual Ideals

Supplement 2. Testing Differences Across Subscales of the Sexual Ideals Measure: Exploratory Analyses Examining Unmet Sexual Ideals Across the Two Factors in Study 1

Supplement 3. Testing Gender Differences Across the Studies

Supplement 4. Testing Partner Interactions Across the Studies

Supplement 5. Study 4: Pilot Study 4A

Supplement 6. References for Supplemental Materials 


\section{References}

Acevedo, B. P., \& Aron, A. (2009). Does a long-term relationship kill romantic love? Review of General Psychology, 13, 59-65.

Ackerman, R. A., Ledermann, T., \& Kenny, D. A. (in prep). Power analysis for the Actor-Partner Interdependence Model.

Aiken, L. S., West, S. G., \& Reno, R. R. (1991). Multiple regression: Testing and interpreting interactions. Sage.

Baumeister, R. F., Catanese, K. R., \& Vohs, K. D. (2001). Is there a gender difference in strength of sex drive? Theoretical views, conceptual distinctions, and a review of relevant evidence. Personality and Social Psychology Review, 5, 242-273.

Beck, J. G. (1995). Hypoactive sexual desire disorder: An overview. Journal of Consulting and Clinical Psychology, 63, $919-927$.

Beck, J. G., Bozman, A. W., \& Qualtrough, T. (1991). The experience of sexual desire:

Psychological correlates in a college sample. Journal of Sex Research, 28, 443-456.

Bentler, P. (1990). Comparative fit indexes in structural models. Psychological Bulletin, 107, 238-246.

Bentler, P. M., \& Bonett, D. G. (1980). Significance tests and goodness of fit in the analysis of covariance structures. Psychological Bulletin, 88, 588-606.

Berinsky, A.J., Margolis, M.F., \& Sances, M.W. (2014). Separating the shirkers from the workers? Making sure respondents pay attention on self-administered surveys. American Journal of Political Science, 58, 739-753.Birnbaum, G. E., \& Reis, H. T. (2006). Women's sexual working models: An evolutionary-attachment perspective. Journal of Sex Research, 43, 328-342. 
Birnbaum, G. E., \& Reis, H. T. (2012). When does responsiveness pique sexual interest? Attachment and sexual desire in initial acquaintanceships. Personality and Social Psychology Bulletin, 38, 946-958.

Birnbaum, G. E., Reis, H. T., Mizrahi, M., Kanat-Maymon, Y., Sass, O., \& Granovski-Milner, C. (2016). Intimately connected: The importance of partner responsiveness for experiencing sexual desire. Journal of Personality and Social Psychology, 111, 530-546.

Blanchflower, D., \& Oswald, A. (2004). Money, sex and happiness. An empirical study. Scandinavian Journal of Economics, 106, 393-415.

Bolger, N., Davis, A., \& Rafaeli, E. (2003). Diary methods: Capturing life as it is lived. Annual Review of Psychology, 54, 579-616.

Bolger, N., \& Laurenceau, J. (2013). Intensive longitudinal methods: An introduction to diary and experience sampling research. New York, NY: Guilford Press.

Braun, V., \& Clarke, V. (2006). Using thematic analysis in psychology. Qualitative Research in Psychology, 3, 77-101.

Browne, M. W., \& Cudeck, R. (1989). Single sample cross-validation indices for covariance structures. Multivariate Behavioral Research, 24, 445-455.

Burke, T. J., \& Young, V. J. (2012). Sexual transformations and intimate behaviors in romantic relationships. Journal of Sex Research, 49, 454-463.

Buss, D. M. (1999). Evolutionary psychology: The new science of the mind. Boston, MA: Allyn and Bacon.

Butzer, B., \& Campbell, L. (2008). Adult attachment, sexual satisfaction, and relationship satisfaction: A study of married couples. Personal Relationships, 15, 141-154. 
Buyukcan-Tetik, A., Campbell, L., Finkenauer, C., Karremans, J. C., \& Kappen, G. (2017). Ideal standards, acceptance, and relationship satisfaction: Latitudes of differential effects. Frontiers in Psychology, 8, 1-11.

Byers, E. S. (2005). Relationship satisfaction and sexual satisfaction: A longitudinal study of individuals in long-term relationships. Journal of Sex Research, 42, 113-118.

Byers, E. S., Demmons, S., \& Lawrance, K. A. (1998). Sexual satisfaction within dating relationships: A test of the interpersonal exchange model of sexual satisfaction. Journal of Social and Personal Relationships, 15, 257-267.

Byers, E. S., \& Heinlein, L. (1989). Predicting initiations and refusals of sexual activities in married and cohabiting heterosexual couples. Journal of Sex Research, 26, 210-231.

Call, V., Sprecher, S., \& Schwartz, P. (1995). The incidence and frequency of marital sex in a national sample. Journal of Marriage and the Family, 57, 639-652.

Campbell, L., Chin, K., \& Stanton, S. C. (2016). Initial evidence that individuals form new relationships with partners that more closely match their ideal preferences. Collabra, 2, $1-7$.

Campbell, L., Overall, N. C., Rubin, H., \& Lackenbauer, S. D. (2013). Inferring a partner's ideal discrepancies: Accuracy, projection, and the communicative role of interpersonal behavior. Journal of Personality and Social Psychology, 105, 217-233.

Campbell, L., Simpson, J. A., Kashy, D. A., \& Fletcher, G. J. O. (2001). Ideal standards, the self, and flexibility of ideals in close relationships. Personality and Social Psychology Bulletin, 27, 447-462. 
Campbell, L., \& Stanton, S. C. E. (2014). The predictive validity of ideal partner preferences in relationship formation: What we know, what we don't know, and why it matters. Social Personality Psychology Compass, 8, 485-494.

Canevello, A., \& Crocker, J. (2010). Creating good relationships: responsiveness, relationship quality, and interpersonal goals. Journal of Personality and Social Psychology, 99, 78106.

Cate, R. M., Lloyd, S. A., \& Long, E. (1988). The role of rewards and fairness in developing premarital relationships. Journal of Marriage and the Family, 50, 443-452.

Cattell, R. B. (1966). The scree test for the number of factors. Multivariate Behavioral Research, $1,245-276$.

Charlot, N., Balzarini, R. N., \& Campbell, L. (2019). The influence of romantic rejection on changes in ideal standards, ideal flexibility, and self-perceived mate value. Social Psychology. Advance online publication.

Clark, M. S., \& Lemay, J. E. P. (2010). Close relationships. In S. T. Fiske, D. T. Gilbert, \& G. Lindzey (Eds.), Handbook of social psychology (Vol. 2, 5th ed., pp. 898-940). Hoboken, NJ: John Wiley \& Sons.

Clark, M. S., \& Mills, J. (2012). A theory of communal (and exchange) relationships. In P. A. M. Van Lange, A. W. Kruglanski, \& E. T. Higgins (Eds.), Handbook of Theories of Social Psychology (pp. 232-250). Thousand Oaks, CA: Sage.

Cohen, J. (1960). A coefficient of agreement for nominal scales. Educational and Psychological Measurement, 20, 37-46.

Comrey, A. L., \& Lee, H. B. (1992). A first course in factor analysis (2nd ed.). Hillsdale, NJ: Erlbaum. 
Conley, T. D., Ziegler, A., Moors, A. C., Matsick, J. L., \& Valentine, B. (2013). A critical examination of popular assumptions about the benefits and outcomes of monogamous relationships. Personality and Social Psychology Review, 17, 124-141.

Cronbach, L. J., \& Furby, L. (1970). How we should measure "change": Or should we? Psychological Bulletin, 74, 68-80.

Csajbók, Z., \& Berkics, M. (2017). Factor, factor, on the whole, who's the best fitting of all?: Factors of mate preferences in a large sample. Personality and Individual Differences, 114, 92-102.

Cupach, W. R., \& Comstock, J. (1990). Satisfaction with sexual communication in marriage: Links to sexual satisfaction and dyadic adjustment. Journal of Social and Personal Relationships, 7, 179-186.

Curran, P.G. (2016). Methods for the detection of carelessly invalid responses in survey data. Journal of Experimental Social Psychology, 66, 4-19.

Day, L., Muise, A., Joel, S., \& Impett, E. A. (2015). To do it or not to do it? How communally motivated people navigate sexual interdependence dilemmas. Personality and Social Psychology Bulletin, 41, 791-804.

Davies, S., Katz, J., \& Jackson, J. (1999). Sexual desire discrepancies: Effects on sexual and relationship satisfaction in heterosexual dating couples. Archives of Sexual Behavior, 28, $553-567$.

De Judicibus, M. A., \& McCabe, M. P. (2002). Psychological factors and the sexuality of pregnant and postpartum women. Journal of Sex Research, 39, 94-103.

Dundon, C. M., \& Rellini, A. H., (2010). More than sexual function: predictors of sexual satisfaction in a sample of women age 40-70. Journal of Sexual Medicine, 7, 896-904. 
Dworkin, S. L., \& O’Sullivan, L. (2005). Actual versus desired initiation patterns among a sample of college men: Tapping disjunctures within traditional male sexual scripts. Journal of Sex Research, 42, 150-158.

Eastwick, P. W., Finkel, E. J., \& Simpson, J. A. (2019). Best practices for testing the predictive validity of ideal partner preference-matching. Personality and Social Psychology Bulletin, 45, 167-181.

Eastwick, P. W., Luchies, L. B., Finkel, E. J., \& Hunt, L. L. (2014). The predictive validity of ideal partner preferences: A review and meta-analysis. Psychological Bulletin, 140, 623665.

Edwards, J. R. (1994). The study of congruence in organizational behavior research: Critique and a proposed alternative. Organizational Behavior and Human Decision Processes, 58, 51100.

Edwards, J. R. (2001). Ten difference score myths. Organizational Research Methods, 4, 265287.

Edwards, J. R., \& Parry, M. E. (1993). On the use of polynomial regression equations as an alternative to difference scores in organizational research. Academy of Management Journal, 36, 1577-1613.

Erdfelder, E., Faul, F., \& Buchner, A. (1996). GPOWER: a general power analysis program. Behavior Research Methods, Instruments, \& Computers, 28, 1-11.

Fabrigar, L. R., Wegener, D. T., MacCallum, R. C., \& Strahan, E. J. (1999). Evaluating the use of exploratory factor analysis in psychological research. Psychological Methods, 4, 272299. 
Faul, F., Erdfelder, E., Buchner, A., \& Lang, A. G. (2009). Statistical power analyses using G* Power 3.1: tests for correlation and regression analyses. Behavior Research Methods, $41,1149-1160$.

Fehr, B. (1988). Prototype analysis of the concepts of love and commitment. Journal of Personality and Social Psychology, 55, 557-579.

Fletcher, G. J., Kerr, P. S., Li, N. P., \& Valentine, K. A. (2014). Predicting romantic interest and decisions in the very early stages of mate selection: standards, accuracy, and sex differences. Personality and Social Psychology Bulletin, 40, 540-550.

Fletcher, G. J., \& Kininmonth, L. A. (1992). Measuring relationship beliefs: An individual differences scale. Journal of Research in Personality, 26, 371-397.

Fletcher, G. J., Overall, N., \& Campbell, L. (revision requested). Reconsidering the "best practices" for testing the predictive validity of ideal standards: A critique of Eastwick, Finkel, and Simpson (2018). Personality and Social Psychology Bulletin.

Fletcher, G. J., \& Simpson, J. (2000). Ideal standards in close relationships. Current Directions in Psychological Science, 3, 102-105.

Fletcher, G. J., Simpson, J. A., Campbell, L., \& Overall, N. C. (2013). The science of intimate relationships (Vol. 323). Malden, MA: Wiley-Blackwell.

Fletcher, G. J., Simpson, J. A., \& Thomas, G. (2000). Ideals, perceptions, and evaluations in early relationship development. Journal of Personality and Social Psychology, 79, 933940.

Fletcher, G. J. O., Simpson, J. A., \& Thomas, G. (2000). The measurement of perceived relationship quality components: A confirmatory factor analytic approach. Personality and Social Psychology Bulletin, 26, 340 - 354. 
Fletcher, G. J., Simpson, J. A., Thomas, G., \& Giles, L. (1999). Ideals in intimate relationships. Journal of Personality and Social Psychology, 76, 72-89.

Fritz, H. L, \& Helgeson, V. S. (1998). Distinctions of unmitigated communion from communion: Self-neglect and overinvolvement with others. Journal of Personality and Social Psychology, 75, 121-140.

Garcia, R. L., Kenny, D. A., \& Ledermann, T. (2015). Moderation in the actor-partner interdependence model. Personal Relationships, 22, 8-29.

Gerlach, T. M., Arslan, R. C., Schultze, T., Reinhard, S. K., \& Penke, L. (2019). Predictive validity and adjustment of ideal partner preferences across the transition into romantic relationships. Journal of Personality and Social Psychology, 116, 313-330.

Gordon, A. M., \& Chen, S. (2016). Do you get where I'm coming from? Perceived understanding buffers against the negative impact of conflict on relationship satisfaction. Journal of Personality and Social Psychology, 110, 239-260.

Hendrick, S. S. (1988). A generic measure of relationship satisfaction. Journal of Marriage and the Family, 50, 93-98.

Henry, R. G., \& Miller, R. B. (2004). Marital problems occurring in midlife: Implications for couples therapists. American Journal of Family Therapy, 32, 405-417.

Herbenick, D., Mullinax, M., \& Mark, K. (2014). Sexual desire discrepancy as a feature, not a bug, of long-term relationships: Women's self-reported strategies for modulating sexual desire. Journal of Sexual Medicine, 11, 2196-2206.

Higgins, E. T. (1987). Self-discrepancy: A theory relating self and affect. Psychological Review, 94, 319-340. 
Higgins, E. T., Klein, R., \& Strauman, T. (1985). Self-concept discrepancy theory: A psychological model for distinguishing among different aspects of depression and anxiety. Social Cognition, 3, 51-76.

Hira, N. S., \& Overall, N. C. (2011). Improving intimate relationships: Targeting the partner versus changing the self. Journal of Social and Personal Relationships, 28, 610-633.

Hirschfeld, G, \& von Brachel, R. (2014). Multiple-group confirmatory factor analysis in R - a tutorial in measurement invariance with continuous and ordinal indicators. Practical Assessment, Research \& Evaluation, 19, 1-12.

Hogue, J. V., Rosen, N. O., Bockaj, A., Impett, E. A., \& Muise, A. (2019). Sexual communal motivation in couples coping with low sexual desire and arousal: Associations with sexual goals, desire and satisfaction. PlosOne, 14: e0219768.

Horn, J. L. (1965). A rationale and test for the number of factors in factor analysis. Psychometrika, 30, 179-185.

Hu, L., \& Bentler, P. M. (1999). Cutoff criteria for fit indexes in covariance structure analysis: Conventional criteria versus new alternatives. Structural Equation Modeling, 6, 1-55.

Humberg, S., Nestler, S., \& Back, M. D. (2018). Response surface analysis in personality and social psychology: Checklist and clarifications for the case of congruence hypotheses. Social Psychological and Personality Science, 10, 409-419.

Impett, E. A., \& Muise, A. (in press). Relationships and sexuality: You reap what you sow in the bedroom. In D. Schoebi \& B. Campos (Eds.), Close relationships: Pathways to strong bonds. Abingdon, UK: Routledge. 
Impett, E. A., Muise, A., \& Harasymchuk, C. (2018). Giving in the bedroom: The costs and benefits of responding to a partner's sexual needs in daily life. Journal of Social and Personal Relationships, 36, 2455-2473.

Impett, E. A., \& Peplau, L. A. (2003). Sexual compliance: Gender, motivational, and relationship perspectives. Journal of Sex Research, 40, 87-100.

Impett, E. A., Peplau, L. A., \& Gable, S. L. (2005). Approach and avoidance sexual motives: Implications for personal and interpersonal well-being. Personal Relationships, 12, 465482.

Joreskog, K. G., \& Sorbom, D. (1984). LISREL 6: User's guide. Mooresville, IN: Scientific Software International.

Karney, B. R., \& Bradbury, T. N. (1995). The longitudinal course of marital quality and stability: A review of theory, methods, and research. Psychological Bulletin, 118, 3-34.

Katz, J., \& Tirone, V. (2009). Women's sexual compliance with male dating partners:

Associations with investment in ideal womanhood and romantic well-being. Sex Roles, $60,347-356$.

Kelley, D. L., \& Burgoon, J. K. (1991). Understanding marital satisfaction and couple type as functions of relational expectations. Human Communication Research, 18, 40-69.

Kelley, H. H., \& Thibault, J. W. (1978). Interpersonal relations: A theory of interdependence. New York, NY: Wiley.

Kenny, D. A., \& Ackerman, R. A. (2019). Power analysis for the Actor-Partner Interdependence Model. Retrieved from https://robert-ackerman.shinyapps.io/APIMPowerR/

Kenny, D. A., Kashy, D. A., \& Cook, W. L. (2006). The analysis of dyadic data. New York, NY: Guilford. 
Kim, J., Muise, A., \& Impett, E. A. (2018). The relationship implications of rejecting a partner for sex kindly versus having sex reluctantly. Journal of Social and Personal Relationships, 35, 485-508.

Kim, J. J., Muise, A., Sakaluk, J. K., Rosen, N. O., \& Impett, E. A. (2020). When tonight is not the night: Sexual rejection and satisfaction in romantic relationships. Personality and Social Psychology Bulletin. Advance online publication.

Kleiman, E. (2019). Power Curves for Multi-level Studies. Retrieved from https://kleimanlab.org/power-curves/

Klusmann, D. (2002). Sexual motivation and the duration of partnership. Archives of Sexual Behavior, 31, 275-287.

Kogan, A., Impett, E., Oveis, C., Hui, B., Gordon, A., \& Keltner, D. (2010). When giving feels good: The intrinsic benefits of sacrifice in romantic relationships for the communally motivated. Psychological Science, 21, 1918-1924.

Kohut, T., Balzarini, R. N., Fisher, W. A., \& Campbell, L. (2018). Pornography’s associations with open sexual communication and relationship closeness vary as a function of dyadic patterns of pornography use within heterosexual relationships. Journal of Social and Personal Relationships, 35, 655-676.

Laurenceau, J. P., Barrett, L. F., \& Pietromonaco, P. R. (1998). Intimacy as an interpersonal process: The importance of self-disclosure, partner disclosure, and perceived partner responsiveness in interpersonal exchanges. Journal of Personality and Social Psychology, 74, 1238-1251. 
Lawrance, K., \& Byers, E. S. (1998). Interpersonal exchange model of sexual satisfaction questionnaire. In C. M. Davis, W. L. Yarber, R. Bauserman, G. Schreer, \& S. L. Davis (Eds.), Sexuality-related measures: A compendium (2nd ed.). Thousand Oaks, CA: Sage.

Le, B. M., Impett, E. A., Lemay, E. P., Jr., Muise, A., \& Tskhay, K. O. (2018). Communal motivation and well-being in interpersonal relationships: An integrative review and metaanalysis. Psychological Bulletin, 144, 1-25.

Lemay, E. P., Clark, M. S., \& Feeney, B. C. (2007). Projection of responsiveness to needs and the construction of satisfying communal relationships. Journal of Personality and Social Psychology, 92, 834-853.

Little, T. D. (2013). Longitudinal Structural Equation Modeling. New York, NY: Guilford Press. Litzinger, S., \& Gordon, K. C. (2005). Exploring relationships among communication, sexual satisfaction, and marital satisfaction. Journal of Sex and Marital Therapy, 31, 409-424.

Maisel, N. C, \& Gable, S. L. (2009). The paradox of received social support: The importance of responsiveness. Psychological Science, 20, 928-932.

Manne, S., Kashy, D. A., Zaider, T., Lee, D., Kim, I. Y., Heckman, C., . . V Virtue, S. M. (2018). Interpersonal processes and intimacy among men with localized prostate cancer and their partners. Journal of Family Psychology, 32, 664-675.

Mark, K. P. (2012). The relative impact of individual sexual desire and couple desire discrepancy on satisfaction in heterosexual couples. Sexual and Relationship Therapy, 27, 133-146.

Mark, K. P. (2014). The impact of daily sexual desire and daily sexual desire discrepancy on the quality of the sexual experience in couples. Canadian Journal of Human Sexuality, $23,27-33$. 
Mark, K. P., \& Lasslo, J. P. (2018). Maintaining sexual desire in long-term relationships: A systematic review and conceptual model. Journal of Sex Research, 55, 563-581.

Mark, K. P., \& Murray, S. H. (2012). Gender differences in desire discrepancy as a predictor of sexual and relationship satisfaction in a college sample of heterosexual romantic relationships. Journal of Sex \& Marital Therapy, 38, 198-215.

Maxwell, J. A., Joel, S., Peetz, J., MacDonald, G., \& Baucom, B. (2019). Expectations drive perceptions of how much effort a romantic partner puts into the relationship from week to week. Manuscript in preparation.

Maxwell, J. A., Muise, A., MacDonald, G., Day, L. C., Rosen, N. O., \& Impett, E. A. (2017). How implicit theories of sexuality shape sexual and relationship well-being. Journal of Personality and Social Psychology, 112, 238-279.

McHugh, M. L. (2012). Interrater reliability: The kappa statistic. Biochemia Medica, 22, 276282.

McNulty, J. K., \& Fisher, T. D. (2008). Gender differences in response to sexual expectancies and changes in sexual frequency: A short-term longitudinal study of sexual satisfaction in newly married couples. Archives of Sexual Behavior, 37, 229-240.

McNulty, J. K., Wenner, C. A., \& Fisher, T. D. (2016). Longitudinal associations among relationship satisfaction, sexual satisfaction, and frequency of sex in early marriage. Archives of Sexual Behavior, 45, 85-97.

Meltzer, A. L., McNulty, J. K., Jackson, G. L., \& Karney, B. R. (2014). Sex differences in the implications of partner physical attractiveness for the trajectory of marital satisfaction. Journal of Personality and Social Psychology, 106, 418-428. 
Michalos, A. C. (1986). An application of multiple discrepancies theory (MDT) to seniors. Social Indicators Research, 18, 349-373.

Miller, S. A., \& Byers, E. S. (2004). Actual and desired duration of foreplay and intercourse: Discordance and misperceptions within heterosexual couples. The Journal of Sex Research, 41, 301-309.

Miller, R. B., Yorgason, J. B., Sandberg, J. G., \& White, M. B. (2003). Problems that couples bring to therapy: A view across the family life cycle. American Journal of Family Therapy, 31, 395-407.

Mills, J., \& Clark, M. S. (1986). Communications that should lead to perceived exploitation in communal and exchange relationships. Journal of Social and Clinical Psychology, 4, $225-234$.

Muise, A., Bergeron, S., Impett, E. A., \& Rosen, N. O. (2017). The costs and benefits of sexual communal motivation for couples coping with vulvodynia. Health Psychology, 36, 819827.

Muise, A., Harasymchuk, C., Day, L. C., Bacev-Giles, C., Gere, J., \& Impett, E. A. (2019). Broadening your horizons: Self-expanding activities promote desire and satisfaction in established romantic relationships. Journal of Personality and Social Psychology, 116, $237-258$.

Muise, A., \& Impett, E. A. (2015). Good, giving, and game: The relationship benefits of communal sexual responsiveness. Social Psychological \& Personality Science, 6, 164172.

Muise, A., \& Impett, E. A. (2016). Applying theories of communal motivation to sexuality. Social and Personality Psychology Compass, 10, 455- 467. 
Muise, A., \& Impett, E. A. (2019). Sexual communal strength scale. In R. Milhausen, J. K. Sakaluk, T. D. Fisher, C. M. Davis, \& W. Yarber (Eds). The Handbook of SexualityRelated Measures (4 ${ }^{\text {th }}$ ed.). Abingdon, UK: Taylor \& Francis.

Muise, A., Kim, J. J., Impett, E. A., \& Rosen, N. O. (2017). Understanding when a partner is not in the mood: Sexual communal strength in couples transitioning to parenthood. Archives of Sexual Behavior, 46, 1993-2006.

Muise, A., Schimmack, U., \& Impett, E. A. (2016). Sexual frequency predicts greater well-being, but more is not always better. Social Psychological and Personality Science, 7, 295-302.

Murray, S. L., \& Holmes, J. G. (1999). The mental ties that bind: Cognitive structures that predict relationship resilience. Journal of Personality and Social Psychology, 77, 12281244.

Murray, S. L., Holmes, J. G., \& Griffin, D. W. (1996). The self-fulfilling nature of positive illusions in romantic relationships: Love is not blind but prescient. Journal of Personality and Social Psychology, 71, 1155-1180.

Nestler, S., Grimm, K. J., \& Schönbrodt, F. D. (2015). The social consequences and mechanisms of personality: How to analyse longitudinal data from individual, dyadic, round-robin and network designs. European Journal of Personality, 29, 272-295.

O’Connor, B. P. (2000). SPSS and SAS programs for determining the number of components using parallel analysis and Velicer's MAP test. Behavior Research Methods, Instruments, and Computers, 32, 396-402.

Overall, N. C., Fletcher, G. J., \& Simpson, J. A. (2006). Regulation processes in intimate relationships: The role of ideal standards. Journal of Personality and Social Psychology, 91, 662-685. 
Peplau, L. A. (2003). Human sexuality: How do men and women differ?. Current Directions in Psychological Science, 12, 37-40.

Petersen, J. L., \& Hyde, J. S. (2010). A meta-analytic review of research on gender differences in sexuality, 1993-2007. Psychological Bulletin, 136, 21-38.

Prolific. (2014). Retrieved July 2019, from https://www.prolific.co/

Raudenbush, S. W., Bryk, A. S., Cheong, Y. F., \& Congdon, R. T. (2004). HLM 6: Hierarchical linear and nonlinear modeling [Computer software manual]. Chicago, IL: Scientific Software International.

Regan, P. (2000). The role of sexual desire and sexual activity in dating relationships. Social Behavior and Personality, 28, 51-60.

Reis, H. T. (2003). A self-report measure of perceived partner responsiveness. Unpublished manuscript, University of Rochester, Rochester, NY.

Reis, H. T. (2007). Steps toward the ripening of relationship science. Personal Relationships, 14, $1-23$.

Reis, H. T., Lemay, E. P., \& Finkenauer, C. (2017). Toward understanding: The importance of feeling understood in relationships. Social and Personality Psychology Compass, 11, 122.

Reis, H. T., Lee, K. Y., O’Keefe, S. D. \& Clark, M. S. (2018). Perceived partner responsiveness promotes intellectual humility. Journal of Experimental Social Psychology, 79, 21-33.

Risch, G. S., Riley, L. A., \& Lawler, M. G. (2003). Problematic issues in the early years of marriage: Content for premarital education. Journal of Psychology and Theology, 31, 253-269. 
Rosen, N. O., Bailey, K., \& Muise, A. (2018). Degree and direction of sexual desire discrepancy are linked to sexual and relationship satisfaction in couples transitioning to parenthood. Journal of Sex Research, 55, 214-225.

Rosen, R. C. (2000). Prevalence and risk factors of sexual dysfunction in men and women. Current Psychiatry Reports, 2, 189-195.

Rosseel, Y. (2012). lavaan: An R package for structural equation modeling. Journal of Statistical Software, 48, 1-36.

Rusbult, C. E., \& Buunk, B. P. (1993). Commitment processes in close relationships: An interdependence analysis. Journal of Social and Personal Relationships, 10, 175-204.

Rusbult, C. E., Martz, J. M., \& Agnew, C. R. (1998). The investment model scale: Measuring commitment level, satisfaction level, quality of alternatives, and investment size. Personal Relationships, 5, 357-391.

Ruvolo, A. P., \& Veroff, J. (1997). For better or for worse: Real-ideal discrepancies and the marital well-being of newlyweds. Journal of Social and Personal Relationships, 14, 223242.

Sakaluk, J. K., \& Short, S. D. (2017). A methodological review of exploratory factor analysis in sexuality research: Used practices, best practices, and data analysis resources. Journal of Sex Research, 54, 1-9.

Sanford, K. (2003). Expectancies and communication behaviour in marriage: Distinguishing proximal-level effects from distal-level effects. Journal of Social and Personal Relationships, 20, 391-402.

Santilla, P., Wager, I., Witting, K., Harlaar, N., Jern, P., Johansson, A., ... Sandnabba, K. (2007). Discrepancies between sexual desire and sexual activity: Gender differences and 
associations with relationship satisfaction. Journal of Sex and Marital Therapy, 34, 3144.

Schönbrodt, F. D. (2016). RSA: An R package for response surface analysis (version 0.9.10). Retrieved from https://cran.r-project.org/package=RSA.

Schwarz, N., Bless, H., Strack, F., Klumpp, G., Rittenauer-Schatka, H., \& Simons, A. (1991). Ease of retrieval as information: Another look at the availability heuristic. Journal of Personality and Social Psychology, 61, 195-202.

Schwartz, P., \& Young, L. (2009). Sexual satisfaction in committed relationships. Sexuality Research and Social Policy, 6, 1-17.

Segal, N., \& Fraley, R. C. (2016). Broadening the investment model: An intensive longitudinal study on attachment and perceived partner responsiveness in commitment dynamics. Journal of Social and Personal Relationships, 33, 581-599.

Selig, J. P., \& Preacher, K. J. (2008). Monte Carlo method for assessing mediation: An interactive tool for creating confidence intervals for indirect effects [Computer software]. Retrieved from http://quantpsy.org/

Simpson, J. A., \& Campbell, L. (Eds.). (2013). The Oxford handbook of close relationships. Oxford University Press.

Simpson, J. A., Fletcher, G. J. O., \& Campbell, L. (2001). The structure and function of ideal standards in close relationships. In G. J. O Fletcher \& M. Clark (Eds.), Blackwell Handbook of Social Psychology: Interpersonal Processes (pp. 86-106). Oxford, England: Blackwell.

Sprecher, S. (2002). Sexual satisfaction in premarital relationships: Associations with satisfaction, love, commitment, and stability. Journal of Sex Research, 39, 190-196. 
Sutherland, S. E., Rehman, U. S., Fallis, E. E., \& Goodnight, J. A. (2015). Understanding the phenomenon of sexual desire discrepancy in couples. The Canadian Journal of Human Sexuality, 24, 141-150.

Tabachnick, B. G., \& Fidell, L. S. (2012). Using multivariate statistics $\left(6^{\text {th }}\right.$ ed.). Upper Saddle River, NJ: Pearson.

Thibaut, J. W., \& Kelley, H. H. (1959). The social psychology of groups. Oxford, England: Wiley.

Visserman, M. L., Muise, A., Righetti, F., Horne, R. M., \& Impett, E. A. (in prep). Lightening the load: Perceived partner responsiveness fosters more favorable appraisals of relational sacrifices.

Visserman, M. L., Muise, A., Righetti, F., Horne, R. M., \& Impett. E. A. (2019, October). Lightening the load: Perceived partner responsiveness fosters more favorable appraisals of relational sacrifices. Symposium conducted at the Society for Experimental Social Psychology Meeting, Toronto, Ontario, Canada.

Walton, G. M. (2014). The new science of wise psychological interventions. Current Directions in Psychological Science, 23, 73-82.

Webster, G. D., Laurenceau, J.-P., Smith, C. V., Mahaffey, A. L., Bryan, A. D., \& Brunell, A. B. (2015). An investment model of sociosexuality, relationship satisfaction, and commitment: Evidence from dating, engaged, and newlywed couples. Journal of Research in Personality, 55, 112-126.

Willoughby, B. J., Farero, A. M., \& Busby, D. M. (2014). Exploring the effects of sexual desire discrepancy among married couples. Archives of Sexual Behavior, 43, 551-562. 
Willoughby, B. J., \& Vitas, J. (2012). Sexual desire discrepancy: The effect of individual differences in desired and actual sexual frequency on dating couples. Archives of Sexual Behavior, 41, 477-486.

Yeh, H. C., Lorenz, F. O., Wickrama, K. A., Conger, R. D., \& Elder, G. H. (2006). Relationships among sexual satisfaction, marital quality, and marital instability in midlife. Journal of Family Psychology, 20, 339-343.

Zhang, Z., Zyphur, M. J., \& Preacher, K. J. (2009). Testing multilevel mediation using hierarchical linear models: Problems and solutions. Organizational Research Methods, $12,695-719$.

Zilbergeld, B., \& Ellison, C. R. (1980). Desire discrepancies and arousal problems in sex therapy. In Leiblum, S. R., \& Pervin, L. A. (Eds.), Principles and Practice of Sex Therapy. New York, NY: Guilford Press. 